\title{
Finite determinacy of matrices over local rings. Tangent modules to the miniversal deformation for $R$-linear group actions.
}

\author{
Genrich Belitskii and Dmitry Kerner
}

\begin{abstract}
We consider matrices with entries in a local ring, $\operatorname{Mat}_{m \times n}(R)$. Fix a group action, $G \circlearrowright M_{m \times n}(R)$, and a subset of allowed deformations, $\Sigma \subseteq M a t_{m \times n}(R)$. The standard question in Singularity Theory is the finite- $(\Sigma, G)$-determinacy of matrices. Finite determinacy implies algebraizability and is equivalent to a stronger notion: stable algebraizability.

In our previous work this determinacy question was reduced to the study of the tangent spaces $T_{(\Sigma, A)}, T_{(G A, A)}$, and their quotient, the tangent module to the miniversal deformation, $T_{(\Sigma, G, A)}^{1}=T_{(\Sigma, A)} T_{(G A, A)}$. In particular, the order of determinacy is controlled by the annihilator of this tangent module, $\operatorname{ann}\left(T_{(\Sigma, G, A)}^{1}\right)$.

In this work we study this tangent module for the group action $G L(m, R) \times G L(n, R) \circlearrowright M_{m \times n}(R)$ and various natural subgroups of it.

We obtain ready-to-use criteria of determinacy for deformations of (embedded) modules, (skew-)symmetric forms, filtered modules, filtered morphisms of filtered modules, chains of modules and others.
\end{abstract}

\section{INTRODUCTION}

11.' Setup. Let $R$ be a (commutative, associative) local ring over some base field $\mathbb{k}$ of zero characteristic. (The simplest examples are $\mathbb{k}[[\underline{x}]], \mathbb{C}\{\underline{x}\}$, and $C^{\infty}\left(\mathbb{R}^{p}, 0\right)$.) Denote by $\mathfrak{m} \subset R$ the maximal ideal and by $\operatorname{Mat}_{m \times n}(R)$ the $R$-module of $m \times n$ matrices with entries in $R$. We always assume $m \leq n$, otherwise one can transpose the matrix.

Various groups act on $M a t_{m \times n}(R)$, we consider mostly the following actions.

Exámple 1.1. Let $A \in \operatorname{Mat}_{m \times n}(R)$.

'The left multiplications $G_{l}:=G L(m, R)$, the right and the two-sided multiplications $G_{r}:=G L(n, R), G_{l r}:=G_{l} \times G_{r}$. Matrices considered up to $G_{r}$-transformations correspond to the embedded modules, $\operatorname{Im}(A) \subset R^{\oplus m}$. Matrices considered up to $G_{l r}$-transformations correspond to the non-embedded modules, $\operatorname{coker}(A)=R^{\oplus m} / \operatorname{Im}(A)$.

ii. The congruence, $G_{\text {congr }}=G L(m, R) \circlearrowright M a t_{m \times m}(R)$, acts by $A \rightarrow U A U^{t}$. Matrices considered up to the congruence $\checkmark$ 'correspond to the bilinear/symmetric/skew-symmetric forms. The conjugation, $G_{\text {conj }}=G L(m, R) \circlearrowright M a t_{m \times m}(R)$, acts by $A \rightarrow U A U^{-1}$. This action is important in representation theory.

iii. 'Fix some ordered compositions, $m=\sum_{i=1}^{k} m_{i}, n=\sum_{j=1}^{k} n_{j}$. Take the corresponding block-structure on the matrix, i.e. $A=$ $\left\{A_{i j}\right\}_{1 \leq i, j \leq k}, A_{i j} \in \operatorname{Mat}_{m_{i} \times n_{j}}(R)$. Denote by $\operatorname{Mat}_{\left\{m_{i}\right\} \times\left\{n_{j}\right\}}^{u p}(R)$ the set of the upper-block-triangular matrices, i.e. $A_{i j}=\mathbb{O}$ 'for $i>j$. Thus $\operatorname{Mat}_{\left\{m_{i}\right\} \times\left\{n_{j}\right\}}^{u p}(R)$ is a free direct summand of $M a t_{m \times n}(R)$. Accordingly we consider the groups

$$
G_{l}^{u p}:=G_{l} \cap M a t_{\left\{m_{i}\right\} \times\left\{m_{j}\right\}}^{u p}(R), \quad G_{r}^{u p}:=G_{r} \cap M a t_{\left\{n_{i}\right\} \times\left\{n_{j}\right\}}^{u p}(R) \text { and } G_{l r}^{u p}:=G_{l}^{u p} \times G_{r}^{u p} .
$$

The upper-block-triangular matrices considered up to the $G_{r}^{u p}$-equivalence correspond to the submodules of $R^{\oplus m}$ filtered by ' chains of direct summands of $R^{\oplus m}$. The upper-block-triangular matrices considered up to the $G_{l r}^{u p}$-equivalence correspond to filtered homomorphisms of filtered free modules. (See $\S 4.3$ for more detail.)

We study deformations of matrices. In applications one often deforms a matrix not inside the whole $M a t_{m \times n}(R)$ but only inside a "deformation subspace" (a subset of prescribed deformations), $A \rightsquigarrow A+B, A+B \in \Sigma \subseteq M a t_{m \times n}(R)$.

In this paper the subset $\Sigma-\{A\} \subseteq M a t_{m \times n}(R)$ is a submodule. Besides the trivial choice $\Sigma=M a t_{m \times n}(R)$, we mostly work with the following submodules.

Example 1.2. i. The congruence of (skew-)symmetric matrices, $A \stackrel{G_{\text {congr }}}{\sim} U A U^{t}, U \in G L(m, R)$, preserves the (skew-) symmetry. Thus it is natural to deform the matrix by the (skew-)symmetric matrices only, i.e. $\Sigma=M a t_{m \times m}^{s y m}(R)$ or $\Sigma=$ $M a t_{m \times m}^{\text {skew-sym }}(R)$.

ii. Similarly, it is natural to deform an upper-block-triangular matrix only inside $\operatorname{Mat}_{\left\{m_{i}\right\} \times\left\{n_{j}\right\}}^{u p}(R)$.

iii. Sometimes one deforms only by "higher order terms", e.g. $\Sigma=\{A\}+M a t_{m \times m}(J)$ or $\Sigma=\{A\}+M a t_{m \times m}^{\text {sym }}(J)$, for some ideal $J \subset R$.

Recall that over a local ring any matrix is $G_{l r}$-equivalent to a block-diagonal, $A \stackrel{G_{l r}}{\sim} \mathbb{I} \oplus \widetilde{A}$, where all the entries of $\widetilde{A}$ lie in the maximal ideal $\mathfrak{m}$, i.e. vanish at the origin of $\operatorname{Spec}(R)$. Similar statements hold for (skew-) symmetric matrices with respect to

Date: April 25, 2019.

2000 Mathematics Subject Classification. Primary 58K40, 58K50 Secondary 32A19, 14B07, 15 A21.

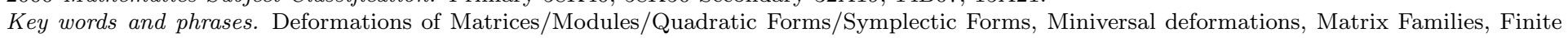
Determinacy, Matrix Singularities, Sufficiency of Jets, Algebraization, Matrix Preservers over Rings.

D.K. was supported by the Israel Science Foundation (grant No. 844/14). 
$G_{\text {congr }}$, see Proposition 3.1. This splitting is natural in various senses and is standard in Commutative Algebra, Singularity Theory and other fields. Often it is $\widetilde{A}$ that carries all the essential information. Therefore we often assume $\left.A\right|_{0}=\mathbb{O}$, i.e. $A \in M a t_{m \times n}(\mathfrak{m})$.

1.2. The tangent spaces. Fix an action $G \circlearrowright M a t_{m \times n}(R)$, a deformation space $\Sigma \subseteq M a t_{m \times n}(R)$, and a matrix $A \in \Sigma$. We assume that the orbit $G A$ and the deformation space possess well defined tangent spaces at $A$, which are $R$-modules. While the general definitions/conditions are technical (see [B.K.1]), in all the cases relevant to our paper the tangent spaces are "the expected ones", see $\S 3.8$.

The standard approach of deformation theory is to establish the existence of the miniversal (semi-universal) deformation and, when the later exists, to understand/to compute its tangent cone. Accordingly one passes from the study of the germs $(G A, A) \subseteq(\Sigma, A)$, to the study of the tangent spaces, $T_{(G A, A)} \subseteq T_{(\Sigma, A)}$.

Much of the information about the deformation problem is encoded in the quotient module

$$
T_{(\Sigma, G, A)}^{1}:=T_{(\Sigma, A)} /_{(G A, A)} .
$$

This $R$-module is the tangent space to the miniversal deformation, when the later exists and is smooth. It often controls the deformation theory of modules, (skew-)symmetric forms and other objects. In Singularity Theory such a module is known as the Tjurina algebra for the contact equivalence, and the Milnor algebra for the right equivalence, [AGLV-1].

This module $T_{(\Sigma, G, A)}^{1}$ is the main object of our study.

1.3. Finite and infinite determinacy. The $(\Sigma, G)$-order of determinacy of $A$ is the minimal number $k \leq \infty$ satisfying: if $A, A_{1} \in \Sigma$ and $\operatorname{jet}_{k}(A)=\operatorname{jet}_{k}\left(A_{1}\right)$ then $A_{1} \in G A$. Here $\operatorname{jet}_{k}$ is the projection $\operatorname{Mat}_{m \times n}(R) \stackrel{\text { jet }_{k}}{\rightarrow} M a t_{m \times n}\left(R / m^{k+1}\right)$. More precisely: Definition 1.3. $\operatorname{ord}_{G}^{\Sigma}(A):=\min \left\{k \mid \Sigma \cap\left(\{A\}+M a t_{m \times n}\left(\mathfrak{m}^{k+1}\right)\right) \subseteq G A\right\} \leq \infty$.

(We assume that the minimum is taken here over a non-empty set.)

If $\operatorname{ord}_{G}^{\Sigma}(A)<\infty$ then $A$ is called finitely-( $\left.\Sigma, G\right)$-determined. In words: $A$ is determined (up to $G$-equivalence) by a finite number of terms of its Taylor expansion at the origin.

Usually $R$ contains a polynomial subring, $\mathbb{k}[x] /$, with the property $\mathfrak{m}=R \cdot(\underline{x})$, i.e. the elements of $R$ are power series $/$ functions in $\underline{x}$. Then an immediate consequence of finite determinacy is the algebraization: $A$ is $G$-equivalent to a matrix of polynomials in $\underline{x}$. Moreover, the order of determinacy gives an upper bound on the degrees of polynomials. We show that the finite determinacy is equivalent to the stable algebraizability (a strengthening of the ordinary algebraizability), see $\S 2.7$ and $\S 3.12$.

If $\operatorname{ord}_{G}^{\Sigma}(A)=\infty$ the matrix is called infinitely determined. This condition is empty when $\mathfrak{m}^{\infty}:=\bigcap_{k>0} \mathfrak{m}^{k}=\{0\}$. But for some rings $\{0\} \neq \mathfrak{m}^{\infty} \subset R$, then even the infinite determinacy is a non-trivial property. (The main example is the ring of germs of smooth functions, $R=C^{\infty}\left(\mathbb{R}^{p}, 0\right)$, and its sub-quotients.) In this case $A$ is determined (up to $G$-equivalence) by its image under the $\mathfrak{m}$-adic completion, $\widehat{A} \in \operatorname{Mat}_{m \times n}(\widehat{R})$, i.e. its full Taylor expansion at the origin.

The finite and infinite determinacies are fundamental notions of Singularity Theory. For Algebraic Geometry/Commutative Algebra the finite determinacy means (roughly) "the deformation theory is essentially finite dimensional". More generally, as the determinacy expresses the "minimal stability", it is important in any area dealing with matrices over rings (or matrix families or matrices depending on parameters).

See $\S 2.9$ for further motivation and $\S 2.10$ for a brief overview and the relation of our work to the known results.

In [B.K.1] we have reduced the study of determinacy to the understanding of the support/annihilator of the module $T_{(G, \Sigma, A)}^{1}$. The matrix $A$ is (in)finitely determined iff $\mathfrak{m}^{N} T_{(\Sigma, A)} \subseteq T_{(G A, A)}$ for some $N \leq \infty$, equivalently $\mathfrak{m}^{N} T_{(G, \Sigma, A)}^{1}=\{0\}$ or $\mathfrak{m}^{N} \subseteq$ $\operatorname{ann}\left(T_{(G, \Sigma, A)}^{1}\right)$. The order of determinacy is fixed by the annihilator $\operatorname{ann}\left(T_{(G, \Sigma, A)}^{1}\right)$, see $\S 3.11$ for more detail.

For Artinian rings the finite determinacy is not interesting, e.g. if $\mathfrak{m}^{N+1}=\{0\}$ then any matrix over $R$ is trivially $N$ determined. Therefore in this paper we often assume that $R$ is not Artinian, i.e. $\mathfrak{m}^{N} \neq\{0\}$ for any $N<\infty$. Geometrically this means: $\operatorname{dim}(\operatorname{Spec}(R))>0$.

1.4. Contents of the paper. The results of [B.K.1] reduce the study of (in)finite determinacy to the computation of the support of $T_{(\Sigma, G, A)}^{1}$, i.e. the annihilator ideal $\operatorname{ann}\left(T_{(\Sigma, G, A)}^{1}\right)$. In the current paper we study the module $T_{(\Sigma, G, A)}^{1}$ and its support for the $R$-linear actions $G \circlearrowright M_{m \times n}(R)$ of example 1.1 .

One could begin from a completely general (set-theoretic) action $G \circlearrowright M a t_{m \times n}(R)$. However, if an action $G \circlearrowright M a t_{m \times n}(R)$ is $R$-linear (though does not necessarily preserve the row/column structure) and sends the degenerate matrices to degenerate (quite a reasonable assumption!) then $G \subseteq G_{l r}$. This property is proved in $\S 3.6$, it belongs to the area of "preserver problems on matrices", exhaustively studied for matrices over fields, but less known for matrices over rings. Therefore example 1.1 contains the main "reasonable" cases.

We compute (or at least bound) the annihilator $\operatorname{ann}\left(T_{(\Sigma, G, A)}^{1}\right)$, the main results are stated in $\S 2$. This gives the ready-to-use criteria to determine (or at least to bound) $\operatorname{ord}_{G}^{\Sigma}(A)$ for various group-actions. We observe the standard dichotomy: for a given data $\left(m, n, \operatorname{dim}(R), G \circlearrowright \Sigma \subseteq \operatorname{Mat}_{m \times n}(R)\right)$ either there are no finitely determined matrices in $M a t_{m \times n}(\mathfrak{m}) \cap \Sigma$, or the generic finite determinacy holds. 
The proofs of these results, corollaries/examples and further developments are in $\S 4$. In many cases we strengthen (generalize and quantify) both the classical and the relatively recent results.

In $\S 3$ we collect the needed background material: determinantal ideals and Pfaffian ideals, annihilator-of-cokernel, the integral closure of ideals and modules, the matrix preservers, tangent spaces to the group orbits, approximation properties and the main result of [B.K.1].

1.5. Acknowledgements. Many thanks to A.Fernandez-Boix, R.-O. Buchweitz, V. Grandjean, G.-M. Greuel, V. Kodiyalam and M. Leyenson for helpful/informative discussions and to L. Molnár, V.V. Sergeichuk for the much needed references on the "matrix preservers" problems. Finally, we thank the anonymous referee for careful reading of the paper and valuable suggestions.

\section{The MAIN Results}

Below we state the main theorems and corollaries. Their proofs and examples are in $\S 4$.

Unless stated otherwise, $R$ is just a commutative unital ring, not necessarily local or Noetherian.

2.1. Notations and Conventions. We denote by $\underline{x}$ the multivariable $x_{1}, \ldots, x_{p}$.

Let $A \in \operatorname{Mat}_{m \times n}(R)$ and denote by $I_{j}(A)$ the determinantal ideal of all the $j \times j$ minors. Denote by ann.coker $(A)$ the annihilator-of-cokernel ideal of the homomorphism of free modules $R^{\oplus n} \stackrel{A}{\rightarrow} R^{\oplus m}$. The properties/relation/computability of these ideals are discussed in $\S 3.2$.

We often use the ideal quotient, $(I: J)=\{f \in R \mid f J \subseteq I\}$ and the integral closure of ideals $I \subseteq \bar{I}$, see $\S 3.4$. Sometimes we write $I: J$ instead of $(I: J)$, to avoid multiple-brackets formulas.

Sometimes we take the $\mathfrak{m}$-adic completion, $R \rightarrow \widehat{R}$, this induces the completion map $\operatorname{Mat}_{m \times n}(R) \rightarrow \operatorname{Mat}_{m \times n}(\widehat{R})$, denote by $\widehat{A}$ the image of $A$.

Suppose $R$ is local and $J \supseteq \mathfrak{m}^{\infty}$. The Loewy length, $l l_{R}(J) \leq \infty$, is the minimal number $N \leq \infty$ that satisfies: $J \supseteq \mathfrak{m}^{N}$. This number also equals the degree of the socle of the quotient module $R / J$. For $R=\mathbb{k}[[\underline{x}]]$ we have yet another expression, via the Castelnuovo-Mumford regularity, $l l_{R}(J)=\operatorname{reg}(R / J)+1$, see [Eisenbud, exercise 20.18].

For the applications to the (in)finite determinacy we assume (in such cases we state this explicitly):

$(R, \mathfrak{m})$ is a local ring over a field $\mathbb{k}$ of zero characteristic, and

either $R$ is Noetherian or the $\mathfrak{m}$-adic completion map is surjective, $R \rightarrow \widehat{R}$, and $\widehat{R}$ is Noetherian.

In this case by $\operatorname{dim}(R)$ we mean $\operatorname{dim}(\widehat{R})$.

Many rings satisfy this condition, e.g. $\mathbb{k}[\underline{x}\rangle / I, \mathbb{k}\langle\underline{x}\rangle / I$ (algebraic power series), $\mathbb{k}\{\underline{x}\} / I$ (convergent power series), $\mathbb{k}[[\underline{x}]\} / I$ and $C^{\infty}\left(\mathbb{R}^{p}, 0\right)$. (For the later see see $\S 3.10$.) But the ring $C^{r}\left(\mathbb{R}^{p}, 0\right)$, of r-times continuously differentiable germs, for $0<r<\infty$, does not satisfy condition (3). Its completion is not Noetherian, e.g. because the ideal $\mathfrak{m}^{r+0^{+}}:=\left\{g \cdot \mathfrak{m}^{r} \mid g \in C^{0}\left(\mathbb{R}^{p}, 0\right), g(0)=0\right\}$ is not finitely generated, and neither is its image in $\widehat{C^{r}\left(\widehat{\mathbb{R}^{p}}, 0\right)}$.

In some cases we assume that the ring $R$ is a principal ideal domain (PID) or a discrete valuation ring (DVR) or a unique factorization domain (UFD), then we state this explicitly.

2.1.1. Generic properties. In this paper the genericity is always used in the following sense of [Tougeron]. Fix some "parameter space" $M$, typically a finitely generated $R$-module, suppose the $j e t_{d}$-projections $\left\{R \stackrel{\text { jet }_{d}}{\rightarrow} R / \mathrm{m}^{d+1}\right\}_{d \in \mathbb{N}}$ induce the projections $M \stackrel{\text { jet }}{\rightarrow}$ jet $_{d}(M)$. We assume that all $\operatorname{jet}_{d}(M)$ are algebraic subschemes over $\mathbb{k}$. A property $\mathcal{P}$ is said to hold generically in $M$ if it holds for all points of $M$ lying in the complement to a subset $X \subset M$ which is "of infinite codimension". Namely, for any $d$ the projection of $X$ lies inside some algebraic subschemes, $\operatorname{jet}_{d}(X) \subseteq Y_{d} \subset \operatorname{jet}_{d}(M)$, such that $\operatorname{codim}_{\text {jet }}(M)\left(Y_{d}\right) \rightarrow \infty$.

Definition 2.1. We say that the generic finite determinacy holds for a given action $G \circlearrowright \Sigma$ if for any $A \in \Sigma$, any number $N<\infty$ and the generic matrix $B \in \operatorname{Mat}_{m \times n}\left(\mathfrak{m}^{N}\right)$, such that $A+B \in \Sigma$, the matrix $A+B$ is finitely determined.

Equivalently: the $\mathfrak{m}$-adic closure of the set of finitely-determined matrices is the whole space $\Sigma$.

Recall that in singularity theory the dimension of the vector space $T_{(\Sigma, G, z)}^{1}$ is called the Tjurina number, for a given deformation scenario $(\Sigma, G, A)$. The finite determinacy means the finiteness of the relevant Tjurina number. The genericity of finite determinacy means: the stratum of matrices whose Tjurina number is infinite, $\Sigma_{\tau=\infty}^{G} \subset \Sigma$, is itself of infinite codimension.

\subsection{The criteria for the $G_{r}, G_{l}, G_{l r}$ actions of Example 1.1.}

Theorem 2.2. Let $A \in \Sigma=\operatorname{Mat}_{m \times n}(R)$, with $m \leq n$.

(1) $T_{\left(\Sigma, G_{r}, A\right)}^{1}=(\operatorname{coker}(A))^{\oplus n}$ and therefore ann $\left(T_{\left(\Sigma, G_{r}, A\right)}^{1}\right)=\operatorname{ann.coker}(A)$.

(2) If $m<n$ then ann $\left(T_{\left(\Sigma, G_{l}, A\right)}^{1}\right)=\{0\}$. If $m=n$ then ann $\left(T_{\left(\Sigma, G_{l}, A\right)}^{1}\right)=\operatorname{ann} . \operatorname{coker}(A)$.

(3) $\operatorname{ann}\left(T_{\left(\Sigma, G_{l r}, A\right)}^{1}\right) \supseteq$ ann.coker $(A)$. If $R$ is Noetherian and $I_{m-1}(A) \neq\{0\}$ then

$$
\overline{\operatorname{ann}\left(T_{\left(\Sigma, G_{l r}, A\right)}^{1}\right)} \subseteq \overline{I_{m}(A)}: \overline{I_{m-1}(A)} .
$$


The bounds in part 3 are close and in many cases we have ann $\left(T_{\left(\Sigma, G_{l r}, A\right)}^{1}\right)=\operatorname{ann.coker}(A)$, see examples in $\S 4.1$. In fact, in $\S 4.1$ we prove a slightly stronger statement:

$$
\overline{\operatorname{ann}\left(T_{\left(\Sigma, G_{l r}, A\right)}^{1}\right) \cdot I_{m-1}(A)} \subseteq \overline{I_{m}(A)} .
$$

Theorem 2.2 bounds $\operatorname{ann}\left(T_{\left(\Sigma, G_{l r}, A\right)}^{1}\right)$ in terms of ann.coker $(A)$ and $\overline{I_{m}(A)}: \overline{I_{m-1}(A)}$. These ideals are usually rather small, thus finite- $G_{l r}$-determinacy places severe restrictions on the ring $R$.

Proposition 2.3. Suppose $R$ satisfies condition (3) and let $\Sigma=\operatorname{Mat}_{m \times n}(R)$.

(1) If ann.coker $(A) \supseteq \mathfrak{m}^{\infty}$ then $l_{R}(\operatorname{ann} . \operatorname{coker}(A))-1 \leq \operatorname{ord}_{G_{r}}^{\Sigma}(A) \leq l l_{R}\left(\operatorname{ann} . \operatorname{coker}\left(\left.A\right|_{\mathfrak{m} R} \oplus n\right)\right)-1$.

In particular:

i. If $\operatorname{dim}(R)>(n-m+1)$ then no matrix in $M_{a} t_{m \times n}(\mathfrak{m})$ is finitely-G $G_{r}$-determined.

ii. If $\operatorname{dim}(R) \leq(n-m+1)$ then the generic $G_{r}$-finite determinacy holds.

(2) If $m<n$ then no matrix $A \in \operatorname{Mat}_{m \times n}(\mathfrak{m})$ is finitely-G $G_{l}$-determined. If $m=n$ then ord ${ }_{G_{r}}^{\Sigma}(A)=$ ord $_{G_{l}}^{\Sigma}(A)$.

(3) $\left(G_{l r}\right.$-equivalence)

$i$. If $\operatorname{dim}(R)>(n-m+1)$ then no matrix in $M a t_{m \times n}(\mathfrak{m})$ is finitely-G $G_{l r}$-determined,

ii. Suppose ann.coker $(A) \supseteq \mathfrak{m}^{\infty}$ then

$$
l l_{\widehat{R}}\left(\overline{I_{m}(\widehat{A})}: \overline{I_{m-1}(\widehat{A})}\right)-1 \leq \operatorname{ord}_{G_{l r}}^{\Sigma}(A) \leq l l_{R}\left(\operatorname{ann} \cdot \operatorname{coker}\left(\left.A\right|_{\mathfrak{m} \cdot R^{\oplus n}}\right)\right)-1 .
$$

Here ann.coker $\left(\left.A\right|_{\mathfrak{m} R^{\oplus n}}\right)$ denotes the annihilator-of-cokernel of the restricted map $\mathfrak{m} \cdot R^{\oplus n} \stackrel{A}{\rightarrow} R^{\oplus m}$.

Regarding the notations in part 3.ii: $\widehat{A}$ denotes the image of $A$ under the completion map $R \rightarrow \widehat{R}$. If $R$ is Noetherian then

$$
l l_{\widehat{R}}\left(\overline{I_{m}(\widehat{A})}: \overline{I_{m-1}(\widehat{A})}\right)=l l_{R}\left(\overline{I_{m}(A)}: \overline{I_{m-1}(A)}\right),
$$

see Corollary 3.17, so this number can be computed in either way.

The bounds in this proposition are rather tight, e.g.:

$$
l l_{R}(\operatorname{ann.coker}(A)) \leq l l_{R}\left(\text { ann.coker }\left(\left.A\right|_{\mathfrak{m} R^{\oplus n}}\right)\right) \leq l l_{R}(\operatorname{ann.coker}(A))+1
$$

and also the ideals $\overline{I_{m}(A)}: \overline{I_{m-1}(A)}$, ann.coker $(A)$ are quite close and often coincide. The proofs, examples and other corollaries are given in $\S 4.1$

We remark that part 1 of the proposition implies also criteria for the infinite- $G_{r}$-determinacy, see Corollary 4.8 for the $C^{\infty}\left(\mathbb{R}^{p}, 0\right)$ case.

2.3. The criteria for $G_{\text {congr }}$. Through this subsection we assume $m>1$.

Recall that for skew-symmetric matrices of even size holds $I_{m}(A)=(\operatorname{det}(A))=(P f(A))^{2}$, the square of the Pfaffian ideal of A. Moreover, ann.coker $(A) \supseteq \operatorname{Pf}(A)$, see Proposition 3.5.

For skew-symmetric matrices of odd size: $\operatorname{det}(A)=0$ and ann.coker $(A)=\{0\}$. As the measure of non-degeneracy of such matrices we use the $(m-1)$ 'st Pfaffian ideal

$$
\left(P f_{m-1}(A)\right):=\sum_{i=1}^{m}\left(\operatorname{Pf}\left(A_{\widehat{i}}\right)\right) .
$$

Here $A_{\hat{i}}$ is the $(m-1) \times(m-1)$ block of $A$, obtained by erasing the $i$ th row and column. (Note that $A_{\widehat{i}}$ is skew-symmetric and $\left(\operatorname{Pf}\left(A_{\widehat{i}}\right)\right)^{2}=I_{m-1}\left(A_{\widehat{i}}\right)$.) See $\S 3.3$ for more detail.

Theorem 2.4. Suppose $I_{m-1}(A) \neq\{0\}$.

(1) Let $\Sigma=\operatorname{Mat}_{m \times m}(R)$, suppose $R$ satisfies the condition $(3)$ and $\operatorname{dim}(R)>0$. Then ann $\left(T_{\left(\Sigma, G_{\text {congr }}, A\right)}^{1} \subseteq \mathfrak{m}^{\infty}\right.$. In particular, if $\mathfrak{m}^{\infty}=\{0\}$ then $\operatorname{ann}\left(T_{\left(\Sigma, G_{\text {congr }}, A\right)}^{1}\right)=\{0\}$.

(2) Let $A \in \Sigma=M a t_{m \times m}^{\text {sym }}(R)$ then ann.coker $(A) \subseteq \operatorname{ann}\left(T_{\left.\Sigma, G_{\text {congr }}, A\right)}^{1}\right)$.

Suppose $R$ is Noetherian then ann $\left(T_{\left.\Sigma, G_{\text {congr }}, A\right)}^{1}\right) \subseteq \overline{I_{m}(A)}: \overline{I_{m-1}(A)}$.

(3) $i$. Let $A \in \Sigma=M a t_{m \times m}^{\text {skew-sym }}(R)$ with $m$ even, then ann.coker $(A) \subseteq \operatorname{ann}\left(T_{\left(\Sigma, G_{\text {congr }}, A\right)}^{1}\right)$.

If $R$ is Noetherian then ann $\left(T_{\left(\Sigma, G_{c o n g r}, A\right)}^{1}\right) \subseteq \overline{I_{m}(A)}: \overline{I_{m-1}(A)}$.

ii. Let $A \in \Sigma=M a t_{m \times m}^{\text {skew-sym }}(R)$ with $m$ odd, then $\left(P f_{m-1}(A)\right) \subseteq \operatorname{ann}\left(T_{\left(\Sigma, G_{\text {congr }}, A\right)}^{1}\right)$. If $R$ is Noetherian then ann $\left(T_{\left(\Sigma, G_{\text {congr }}, A\right)}^{1}\right) \subseteq \overline{I_{m-1}(A)}: \overline{I_{m-2}(A)}$.

The bounds in parts 2,3 are rather close and often coincide, see examples in $\S 4.2$.

The bounds on $\operatorname{ann}\left(T_{\left(\Sigma, G_{c o n g r}, A\right)}^{1}\right)$ give immediate applications to the finite determinacy:

Proposition 2.5. Suppose $R$ satisfies the condition (3).

(1) If $\operatorname{dim}(R)>1$ then no $A \in M a t_{m \times m}^{\text {sym }}(\mathfrak{m})$ is finitely- $\left(M_{a t}^{\text {sym }}{ }_{m \times m}^{\text {m }}(R), G_{\text {congr }}\right)$-determined.

Similarly, no $A \in M_{m \times t_{m}^{\text {skew-sym }}}(\mathfrak{m})$ with m-even is finitely-(Mat $\left.{ }_{m \times m}^{\text {skew-sym }}(R), G_{\text {congr }}\right)$-determined.

(1') If $m$ is odd and $\operatorname{dim}(R)>3$ then no $A \in \operatorname{Mat}_{m \times m}^{\text {skew-sym }}(\mathfrak{m})$ is finitely- $\left(M a t_{m \times m}^{\text {skew-sym }}(R), G_{\text {congr }}\right)$-determined.

(1") If $\operatorname{dim}(R)>0$ then no $A \in \operatorname{Mat}_{m \times m}(\mathfrak{m})$ is finitely- $\left(\operatorname{Mat}_{m \times m}(R), G_{\text {congr }}\right)$-determined. 
(2) Suppose $R$ is Henselian.

i. Suppose $\operatorname{dim}(R)=1$ and either $\Sigma=\operatorname{Mat}_{m \times m}^{\text {sym }}(R)$ or $\Sigma=M a t_{m \times m}^{\text {skew-sym }}(R)$ for $m$-even. Let $A \in \Sigma$ and suppose $I_{m}(A) \supsetneq \mathfrak{m}^{\infty}$. Then

$$
l l_{\widehat{R}}\left(\overline{I_{m}(\widehat{A})}: \overline{I_{m-1}(\widehat{A})}\right)-1 \leq \operatorname{ord}_{G_{\text {congr }}}^{\Sigma}(A) \leq l l_{R}(\operatorname{ann.coker}(A)) .
$$

In particular, $A$ is finitely- $\left(\Sigma, G_{\text {congr }}\right)$-determined iff $\operatorname{det}(A)$ is not a zero divisor in $R$. In particular, the generic finite determinacy holds.

ii. Suppose $\operatorname{dim}(R) \leq 3$ and $A \in \Sigma=\operatorname{Mat}_{m \times m}^{\text {skew-sym }}(R)$, for $m$-odd. Suppose moreover $I_{m-1}(A) \supsetneq \mathfrak{m}^{\infty}$. Then

$$
l l_{\widehat{R}}\left(\overline{I_{m-1}(\widehat{A})}: \overline{I_{m-2}(\widehat{A})}\right)-1 \leq \operatorname{ord}_{G_{c o n g r}}^{\Sigma}(A) \leq l l_{R}\left(P f_{m-1}(A)\right) .
$$

In particular, $A$ is finitely- $\left(\Sigma, G_{\text {congr }}\right)$-determined iff either $I_{m-1}(A)=R$ or $I_{m-1}(A) \subset R$ is of height=dim $(R)$, as expected. In particular, the generic finite determinacy holds.

The proofs, examples and further corollaries are in $\S 4.2$.

For the criteria of infinite determinacy of $C^{\infty}\left(\mathbb{R}^{p}, 0\right)$-valued (skew-)symmetric forms see Corollary 4.12.

2.4. Upper-block-triangular matrices. For an upper-block-triangular matrix $A \in \operatorname{Mat}_{\left\{m_{i}\right\} \times\left\{n_{j}\right\}}^{u p}(R)$ take the block-structure, $\left\{A_{i j}\right\}_{1 \leq i, j \leq k}$, as in Example 1.1. The context/motivation for this case, the definitions of $T_{\left(\Sigma, G_{r}^{u p}, A\right)}^{1}, T_{\left(\Sigma, G_{l r}^{u p}, A\right)}^{1}$, their roles, the proofs, examples and corollaries are in $\S 4.3$.

Denote by $\underline{A} \|_{q}$ the sub-matrix of $A$ consisting of the first $\sum_{i=1}^{q} m_{i}$ rows and the first $\sum_{j=1}^{q} n_{j}$ columns. (Thus $\underline{A \|_{1}}$ is the first upper-left block, while $\underline{A \|_{k}}=A$.) We use this notation in part (1) of the theorem:

Theorem 2.6. Let $A \in \Sigma=M a t_{\left\{m_{i}\right\} \times\left\{n_{j}\right\}}^{u p}(R)$.

(1) $\operatorname{ann}\left(T_{\left(\Sigma, G_{r}^{u p}, A\right)}^{1}\right)=\bigcap_{q=1}^{k} \operatorname{ann} \cdot \operatorname{coker}\left(\underline{A}_{q}\right)$.

(2) In particular, $\prod_{q=1}^{k}$ ann.coker $\left(A_{q q}\right) \subseteq \operatorname{ann}\left(T_{\left(\Sigma, G_{r}^{u p}, A\right)}^{1}\right) \subseteq \bigcap_{q=1}^{k} \operatorname{ann} . \operatorname{coker}\left(A_{q q}\right)$.

(3) Suppose $R$ is Noetherian then

$$
\prod_{q=1}^{k} \text { ann.coker }\left(A_{q q}\right) \subseteq \bigcap_{q=1}^{k} \operatorname{ann} . \operatorname{coker}\left(\underline{A \|_{q}}\right) \subseteq \operatorname{ann}\left(T_{\left(\Sigma, G_{l r}^{u p}, A\right)}^{1}\right) \subseteq \bigcap_{q=1}^{k}\left(\overline{I_{m_{q}}\left(A_{q q}\right)}: \overline{I_{m_{q}-1}\left(A_{q q}\right)}\right) .
$$

In part 2 both bounds are sharp, see examples in $\S 4.3$.

2.5. The non-finite determinacy for conjugation. The conjugation action is defined in Example 1.1.

Theorem 2.7. Suppose $R$ satisfies condition (3) and let $A \in M_{m \times m}(R)$.

(1) $\operatorname{ann}\left(T_{\left(M_{m \times m}(R), G_{c o n j}, A\right)}^{1}\right) \subseteq \mathfrak{m}^{\infty}$.

(2) In particular, if $\operatorname{dim}(R)>0$ then there are no finitely-G $G_{c o n j}$-determined matrices.

2.6. Finite determinacy of chains of free modules. As an immediate application of our methods we consider bounded chains (or complexes) of free modules. Take such a chain, $\cdots \stackrel{\phi_{i-1}}{\rightarrow} F_{i} \stackrel{\phi_{i}}{\rightarrow} F_{i-1} \stackrel{\phi_{i-1}}{\rightarrow} \cdots$, fix some bases of $\left\{F_{i}\right\}$, so that the maps are represented by some matrices. The deformations of such a chain are taken up to isomorphisms, induced by the action of the product of groups $\prod_{i} G L\left(F_{i}, R\right)$. We obtain the bounds on the determinacy in $\S 4.5$, quantifying and generalizing theorem 5.8 of [Cutkosky-Srinivasan].

2.7. Algebraization. Suppose $\mathbb{k}[\underline{x}] / I \subseteq R$ and the completion of $R$ at the ideal $(\underline{x})$ is $\widehat{R}=\mathbb{k}[[\underline{x}] / I$. For example, this holds when $R$ is a local ring over a field $\mathbb{k}$, by Cohen Structure Theorem.

A trivial consequence of finite determinacy is the algebraization: the $G$-orbit of a given object contains algebraic representatives (with entries in $\mathbb{k}[\underline{x} / I$ ) and they are obtained by just "cutting the Taylor-tails". Moreover, the order of determinacy gives an upper bound on the possible degrees of polynomials. As we show in $\S 3.12$, finite determinacy is equivalent to stable algebraizability: any higher order deformation of a given object is $G$-equivalent to an algebraic family.

Even if $A$ is not finitely determined, i.e. $\operatorname{ann}\left(T_{(\Sigma, G, A)}^{1}\right)$ does not contain any $\mathfrak{m}^{N}$, one can consider some ideal $J$ such that $J+\operatorname{ann}\left(T_{(\Sigma, G, A)}^{1}\right)$ contains a power of the maximal ideal. Then one gets the "algebraization modulo $J$ ", or the "algebraization with respect to some subset of variables". Therefore our results generalize those of [Elkik], [Kucharz] and many others.

2.8. Relative determinacy/admissible deformations. As we see in Theorems $2.2,2.4,2.6$, the condition ann $\left(T_{(\Sigma, G, A)}^{1}\right) \supseteq \mathfrak{m}^{N}$ can be quite restrictive. For example:

- for the action $G_{l r} \circlearrowright \operatorname{Mat}_{m \times n}(R)$ it means: ann.coker $(A) \supseteq \mathfrak{m}^{N}$, i.e. the module $\operatorname{coker}(A)$ is of finite length. Geometrically: the module is supported only at one point, the origin, i.e. is a skyscraper.

- for the action $G_{c o n g r} \circlearrowright M a t_{m \times m}^{s y m}(R)$ it means: $A$ and its associated quadratic form are non-degenerate on the punctured neighborhood of the origin. 
However, one often needs to deform $A$ not inside the whole $M a t_{m \times n}(R)$, or $\Sigma$, but only by elements of $M a t_{m \times n}(J)$ (for some ideal $J \subset R)$, i.e. the deformation space is $\Sigma^{(J)}:=\Sigma \cap\left(\{A\}+\operatorname{Mat}_{m \times n}(J)\right)$. For example, one studies the determinacy for the action $G_{l r} \circlearrowright \operatorname{Mat}_{m \times n}(J)$ or $G_{\text {congr }} \circlearrowright \operatorname{Mat}_{m \times m}^{\text {sym }}(J)$.

Similarly, starting from a group action, $G \circlearrowright M a t_{m \times n}(R)$, one restricts to the transformations preserving the filtration on $\operatorname{Mat}_{m \times n}(R)$. Often one restricts further, to the subgroup of transformations that are identities modulo some (prescribed) ideal $I \subsetneq R$. More precisely:

$$
G^{(I)}:=\left\{g \in G \mid g \cdot \operatorname{Mat}_{m \times n}(I)=\operatorname{Mat}_{m \times n}(I) \text { and }[g]=[I d] \circlearrowright M a t_{m \times n}(R) / \operatorname{Mat}_{m \times n}(I)\right\} .
$$

For example, for $I=\mathfrak{m}^{k}$ the group $G^{\left(\mathfrak{m}^{k}\right)}$ consists of elements that are identities up to the order $(k-1)$.

Working with $\Sigma^{(J)}$ and $G^{(I)}$ leads to the notion of relative determinacy/admissible deformations. For complex analytic nonisolated hypersurface singularities this was studied in [Pellikaan88], [Pellikaan90], [Siersma83], [Siersma00], [de Jong-van Straten], [Jiang]. For the $C^{\infty}\left(\mathbb{R}^{p}, 0\right)$-version see [Grandjean00], [Grandjean04], [Thilliez], [Cutkosky-Srinivasan].

Our criteria directly adapt to this formulation, see Proposition 4.20 in $\S 4.6$.

\subsection{Remarks.}

2.9.1. Theorems 2.2, 2.4, 2.6 and their corollaries are absolutely explicit, ready-to-use criteria. Their role is similar to the classical Mather theorem (for $\mathbb{k}=\overline{\mathbb{k}}$ ), the transition from:

- "the hypersurface germ is finitely determined iff its miniversal deformation is finite dimensional"

to

- "the hypersurface germ is finitely determined iff it has at most an isolated singularity".

Moreover, we do not just establish the finite determinacy, but give rather tight bounds for the order of determinacy.

To emphasize, in most cases the module $T_{(\Sigma, G, A)}^{1}$ does not admit any explicit/short presentation, e.g. for $T_{\left(M a t_{m \times n}(R), G_{l r}, A\right)}^{1}$ one cannot write anything more explicit than $\operatorname{Ext}_{R}^{1}(\operatorname{coker}(A), \operatorname{coker}(A))$. But the bounds we get are quite explicit and tight, the upper and lower bounds often have the same integral closure.

2.9.2. "Restrictiveness" of the results. As our results read, for many actions there are no finitely determined matrices in $M a t_{m \times n}(\mathfrak{m})$. For example, there are no finitely determined $\mathfrak{m}$-valued quadratic forms when $\operatorname{dim}(R)>1$ (Proposition 2.5$)$. For the action $G_{c o n j} \circlearrowright \operatorname{Mat}_{m \times m}(R)$ the situation is even worse, Theorem 2.7.

Sometimes one insists on having enough finitely determined objects, e.g. one wants the generic finite determinacy in the sense of definition 2.1. The two ways to achieve this are:

- Either to enlarge the group, e.g. to use also the local coordinate changes/automorphisms of the ring, $A u t_{\mathbb{k}}(R)$. Then one considers the action of $G_{l r} \rtimes A u t_{\mathbb{k}}(R), G_{\text {congr }} \rtimes A u t_{\mathbb{k}}(R)$, etc. We do this in [B.K.2].

- Or to restrict the possible deformations, e.g. to deform in a way that preserves the determinantal ideals, $\left\{I_{j}(A)\right\}$, or to preserve the characteristic polynomial for square matrices. We do this in [B.K.3].

2.9.3. A "theoretical" remark. Fix some action $G \circlearrowright \Sigma \subseteq \operatorname{Mat}_{m \times n}(R)$, we assume that $T_{(\Sigma, A)}$ is a free $R$-module. (This holds in our cases.) Take the generating matrix $\mathcal{A}_{G, A}$ of the module $T_{(G A, A)}$ so that $\operatorname{Im}\left(\mathcal{A}_{G, A}\right)=T_{(G A, A)} \subseteq T_{(\Sigma, A)}$. Suppose $\mathcal{A}_{G, A}$ is of size $m_{1} \times n_{1}$, here $m_{1}=\operatorname{rank}\left(T_{(\Sigma, A)}\right)$, then

$$
T_{(\Sigma, G, A)}^{1}=T_{(\Sigma, A)} / T_{(G A, A)}=\operatorname{coker}\left(\mathcal{A}_{G, A}\right), \quad \text { while } \quad T_{\left(M a t_{m_{1} \times n_{1}}(R), G_{r}, \mathcal{A}_{G, A}\right)}^{1}=\operatorname{coker}\left(\mathcal{A}_{G, A}\right)^{\oplus n_{1}} .
$$

(Here on the right $G_{r}=G L\left(n_{1}, R\right)$.) In particular, $\operatorname{ann}\left(T_{(\Sigma, G, A)}^{1}\right)=\operatorname{ann}\left(T_{\left(M_{a t} t_{m_{1} \times n_{1}}(R), G_{r}, \mathcal{A}_{G, A}\right)}^{1}\right)$.

Therefore the study of $T^{1}$ for any $G$ action is "embedded" into the study of $T^{1}$ for the $G_{r}$ action, on the space of bigger size matrices.

2.9.4. Finite determinacy of (non-)embedded modules and (skew-)symmetric forms. The notion of finite determinacy and our results can be stated more algebraically as follows.

The projection $R \stackrel{j t_{k}}{\rightarrow} R / \mathrm{m}^{k+1}$ induces the functor, $\bmod (R) \rightarrow \bmod \left(R / \mathfrak{m}^{k+1}\right)$, defined by $M \rightarrow M / \mathrm{m}^{k+1} M$. This functor is trivially surjective on the objects, by the restriction of scalars. But the functor is not injective on the objects, e.g. if the presentation matrix of $M$ has all its entries in $\mathfrak{m}^{k+1}$ then $\frac{M}{\mathfrak{m}^{k+1}} M$ is a free $R / \mathfrak{m}^{k+1}$ module. Our results give the regions of parameters (the size of presentation matrix, $\operatorname{dim}(R))$ for which the functor is "generically injective on the objects".

Let $M \subseteq R^{\oplus m}$ be a finitely generated $R$-submodule. Fix some set of generators of $M$, combine them into the matrix $A$.

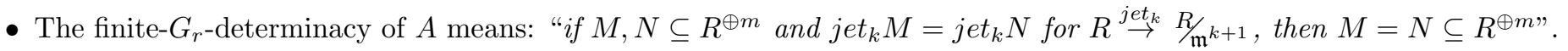

- The finite- $G_{l r}$-determinacy of $A$ means: "if $M, N \subseteq R^{\oplus m}$ and jet ${ }_{k} M=\operatorname{jet}_{k} N$ for $R^{\text {jet }_{k}} R / \mathrm{R}^{k+1}$, then $M=\phi(N) \subseteq R$ ( for some $\phi \in G L(m, R)$ ".

Similarly for (skew-)symmetric forms the finite determinacy means that the form is determined (up to the change of generators) by its $k$ 'th jet for $k \gg 0$. 
2.10. Relation to Singularity Theory. The (in)finite determinacy is a classical notion of Singularity Theory, well studied for functions and maps over the "classical" rings, when $\mathbb{k}=\mathbb{R}$ or $\mathbb{C}$, and $R=\mathbb{k}[[\underline{x}]], \mathbb{k}\{\underline{x}\}, \mathbb{k}[\underline{x}]_{(\underline{x})}, C^{\infty}\left(\mathbb{R}^{p}, 0\right),[\mathrm{Mather}],[\mathrm{Wilson} 81]$, [Wilson82], [Damon84], [Wall81], [AGLV-1], etc.

The generic finite determinacy has been considered in [Wall79], [du Plessis1982], [du Plessis1983].

(For a short discussion of the results on the determinacy of functions/maps see [B.K.1, §2].)

2.10.1. Some known results on "matrix singularities". Some necessary and sufficient conditions for finite determinacy of modules over the ring $C^{\infty}\left(\mathbb{R}^{p}, 0\right)$ were obtained in [Tougeron].

The square matrices over $R=\mathbb{k}\left\{x_{1}, \ldots, x_{p}\right\}$, for $\mathbb{k} \in \mathbb{R}, \mathbb{C}$, and $G=G_{l r} \rtimes A u t(R)$, were considered in [Haslinger], [Bruce], [Bruce-Tari], and further studied in [Bruce-Goryunov-Zakalyukin], [Bruce], [Goryunov-Mond], [Goryunov-Zakalyukin]. In particular, the generic finite determinacy was established and the simple types were classified. We mention also the recent works, [Pereira.10], [Pereira.17], [Ahmed-Ruas].

In [Cutkosky-Srinivasan] they study the finite determinacy of matrices for the actions of $G_{r}, G_{l r}, G_{r} \rtimes A u t_{\mathbb{k}}(R), G_{l r} \rtimes A u t_{\mathbb{k}}(R)$ on matrices over complete local rings. They obtain the qualitative results for the finite determinacy.

In [Damon-Pike.1], [Damon-Pike.2] they study the vanishing topology of matrix singularities, relating these to the free divisors. In [Frühbis-Krüger.Zach] they study the vanishing topology of determinantal singularities.

The study of matrix determinacy in positive characteristic has been initiated in [Greuel-Pham], [Pham].

We emphasize that most of the previous works addressed only the general criteria for the finite determinacy. In our work we directly compute (or at least bound) the order(s) of determinacy.

2.10.2. In our results we see the standard dichotomy: either the finite determinacy is the generic property or there are no finitely determined matrices in $\operatorname{Mat}_{m \times n}(\mathfrak{m}) \cap \Sigma$. This is the analogue of the "nice/bad dimensions" for the determinacy of maps $\operatorname{Maps}\left(\left(\mathbb{k}^{n}, 0\right),\left(\mathbb{k}^{m}, 0\right)\right),[$ AGLV-1, III.1], [Mather].

\section{BACKGRound AND PREPARATIONS}

In this section we collect the standard facts of commutative algebra, thus, unless stated otherwise, $R$ is just a commutative unital ring. We denote the zero matrix by $\mathbb{O}$, the identity matrix by $\mathbb{I}$.

Let $(R, \mathfrak{m})$ be a local $\mathrm{ring}$, with residue field $\mathbb{k}_{k}=R / \mathfrak{m}$. Given $A \in \operatorname{Mat}_{m \times n}(R)$ we sometimes "compute it at the origin", i.e. take its image over the residue field, $\left.A\right|_{0} \in \operatorname{Mat}_{m \times n}(\mathbb{k})$. In particular, $\left.A\right|_{0}=\mathbb{O}$ means $A \in M a t_{m \times n}(\mathfrak{m})$. As $\left.A\right|_{0}$ is a matrix over a field, we take its classical rank, $r=\operatorname{rank}\left(\left.A\right|_{0}\right)$. Recall that the rank of a skew-symmetric matrix is necessarily even.

We denote by $E=\oplus_{i}\left(\begin{array}{cc}0 & 1 \\ -1 & 0\end{array}\right)$ the canonical skew-symmetric matrix, its size (i.e. the number of summands) should be clear from the context.

\subsection{The relevant canonical forms for matrices.}

Proposition 3.1. Let $(R, \mathfrak{m})$ be a local ring.

(1) Let $A \in \operatorname{Mat}_{m \times n}(R)$ then $A \stackrel{G_{l r}}{\sim} \mathbb{1}_{r \times r} \oplus \widetilde{A}$, where $r=\operatorname{rank}\left(\left.A\right|_{0}\right)$ and $\widetilde{A} \in M_{(m-r) \times(n-r)}(\mathfrak{m})$.

(2) Let $A \in \operatorname{Mat}_{m \times m}^{\text {sym }}(R)$ then $A \stackrel{G_{\text {congr }}}{\sim}\left(\oplus_{i} \lambda_{i} \mathbb{I}_{i}\right) \oplus \widetilde{A}$, where $\left\{\lambda_{i} \in R \backslash \mathfrak{m}\right\}_{i}$ and $\widetilde{A} \in \operatorname{Mat}_{(m-r) \times(m-r)}^{\text {sym }}(\mathfrak{m})$.

(3) Let $A \in \operatorname{Mat}_{m \times m}^{\text {skew-sym }}(R)$ then $A \stackrel{G_{\text {congr }}}{\sim}\left(\underset{i}{\oplus} \lambda_{i} E_{i}\right) \oplus \widetilde{A}$, where $\left\{\lambda_{i} \in R \backslash \mathfrak{m}\right\}_{i}$ and $\widetilde{A} \in \operatorname{Mat} t_{(m-r) \times(m-r)}^{\text {skew-sym }}(\mathfrak{m})$.

Part 1 is known in Commutative Algebra e.g. as "passing to the minimal resolution of the module coker $(A)$ ". Similarly, in parts 2,3 we split a (skew-)symmetric form into its regular part (in the canonical form) and the complement - the 'purely degenerate' part.

(Part 2. is proved e.g. in [Birkhoff-MacLane, theorem 3, page 345], for part 3 see [Birkhoff-MacLane, exercise 9, page 347].)

Let $R$ be a discrete valuation ring (DVR) over $\mathbb{k}$. In particular $R$ is local, regular and $\operatorname{dim}(R)=1$. The simplest examples of such rings are the formal power series, $R=\mathbb{k}[[t]]$, or the analytic series, $R=\mathbb{k}\{t\}$, when $\mathbb{k}$ is a normed field. Note that the ring of real-valued smooth function-germs, $C^{\infty}\left(\mathbb{R}^{1}, 0\right)$, is not a DVR. Matrices over DVR often have good canonical forms.

Proposition 3.2. Suppose $R$ is a DVR over $\mathbb{k}$.

(1) For any $A \in \operatorname{Mat}_{m \times n}(R)$ holds: $A \stackrel{G_{l r}}{\sim}\left(\begin{array}{ccccc}\lambda_{1} & 0 & \ldots & \ldots & 0 \\ 0 & \lambda_{2} & 0 & \ldots & \ldots \\ \ldots & \ldots & \ldots & \ldots & \ldots \\ 0 & \ldots & & \lambda_{m} & 0\end{array}\right)$, where $R \supseteq\left(\lambda_{1}\right) \supseteq\left(\lambda_{2}\right) \supseteq \cdots \supseteq\left(\lambda_{m}\right)$.

(2) For any $A \in M a t_{m \times m}^{\text {sym }}(\mathfrak{m})$ holds: $A \stackrel{G_{\text {congr }}}{\sim} \lambda_{i} \mathbb{I}_{i}$, where $R \supseteq\left(\lambda_{1}\right) \supseteq\left(\lambda_{2}\right) \supseteq \cdots \supseteq\left(\lambda_{m}\right)$.

(3) For any $A \in M a t_{m \times m}^{\text {skew-sym }}(\mathfrak{m})$ holds: $A \stackrel{G_{\text {congr }}^{i}}{\sim}\left(\oplus_{i} \lambda_{i} E_{i}\right) \oplus \mathbb{O}$, where $R \supseteq\left(\lambda_{1}\right) \supseteq\left(\lambda_{2}\right) \supseteq \cdots$, while $\mathbb{O}$ is the zero matrix of an appropriate size.

Here part 1 is the Smith normal form. Considering $A$ as a presentation matrix of the $R$-module coker $(A)$, the statement is: "every module over a DVR is a direct sum of cyclic modules". Parts 2,3 read: every (skew-)symmetric form over a DVR splits into the direct sum of rank-one forms. 
While parts 2,3 are standard facts, we did not find an exact reference, thus we sketch a proof. Let $t$ be a uniformizing parameter of $R$. Denote by $p$ the minimal among the vanishing orders of the entries of $A$. Then $t^{-p} A$ is a (skew-)symmetric matrix over $R$ and $\left.t^{-p} A\right|_{0} \neq \mathbb{O}$. Apply Proposition 3.1 to get, in the symmetric case, $A \stackrel{G_{\text {congr }}}{\sim} t^{p}\left(\left(\oplus_{i} \lambda_{i} \mathbb{I}_{i}\right) \oplus \widetilde{A}\right),\left(\right.$ or $A \stackrel{G_{c o n g r}}{\sim} t^{p}\left(\left(\oplus_{i} \lambda_{i} E_{i}\right) \oplus \widetilde{A}\right)$ in the skew-symmetric case). Thus we get the reduction in size and one repeats for $\widetilde{A}$.

3.2. Determinantal ideals and the annihilator-of-cokernel. [Eisenbud, $\S 20$ ] For $0<j \leq m$ and $A \in M a t_{m \times n}(R)$ denote by $I_{j}(A) \subset R$ the $j$ 'th determinantal ideal, generated by all the $j \times j$ minors of $A$. By definition $I_{j \leq 0}(A)=R$ and $I_{j>m}(A)=\{0\}$. When the size of $A$ is not stated explicitly we denote the ideal of maximal minors by $I_{\max }(A)$. The chain of ideals $R=I_{0}(A) \supseteq$ $I_{1}(A) \supseteq \cdots \supseteq I_{m}(A)$ is invariant under the $G_{l r}$-action.

Determinantal ideals behave nicely under ring homomorphisms, for any $R \stackrel{\phi}{\rightarrow} S$ and the induced map $M a t_{m \times n}(R) \stackrel{\phi}{\rightarrow}$ $\operatorname{Mat}_{m \times n}(S)$ one has: $I_{j}(\phi(A))=\phi\left(I_{j}(A)\right)$.

3.2.1. The expected heights. (These will be used later for the generic finite determinacy/bounds on the annihilator.)

If $\left.A\right|_{0}=\mathbb{O}$, i.e. $A \in \operatorname{Mat}_{m \times n}(\mathfrak{m})$, then the height of $I_{j}(A)$ is at most $\min ((m+1-j)(n+1-j)$, $\operatorname{dim}(R))$ and this bound is achieved generically. More precisely, for any $A \in \operatorname{Mat}_{m \times n}(\mathfrak{m})$, any $N>0$ and any generic $B \in M_{m \times n}\left(\mathfrak{m}^{N}\right)$ the height of the ideal $I_{j}(A+B)$ equals $\min ((m+1-j)(n+1-j), \operatorname{dim}(R))$.

For $A \in M a t_{m \times m}^{s y m}(\mathfrak{m})$ the expected height of $I_{j}(A)$ is $\min \left(\left(\begin{array}{c}m-j+2 \\ 2\end{array}\right), \operatorname{dim}(R)\right)$. For $A \in M a t_{m \times m}^{\text {skew-sym }}(\mathfrak{m})$ and $j$ even the expected height of $I_{j}(A)$ is $\min \left(\left(\begin{array}{c}m-j+2 \\ 2\end{array}\right), \operatorname{dim}(R)\right)$. For $A \in M a t_{m \times m}^{s k e w-s y m}(\mathfrak{m})$ and $j$ odd the expected height of $I_{j}(A)$ is $\min \left(\left(\begin{array}{c}m-j+1 \\ 2\end{array}\right), \operatorname{dim}(R)\right)$.

3.2.2. ann.coker $(A)$. A matrix $A \in M a t_{m \times n}(R)$ can be considered as a homomorphism of free modules, its cokernel is an $R$ module as well:

$$
R^{\oplus n} \stackrel{A}{\rightarrow} R^{\oplus m} \rightarrow \operatorname{coker}(A) \rightarrow 0 .
$$

Then one takes the annihilator-of-cokernel ideal:

$$
\operatorname{ann.coker}(A)=\left\{f \in R \mid f \cdot R^{\oplus m} \subseteq \operatorname{Im}(A)\right\}=\operatorname{ann} R^{\oplus m} / \operatorname{Im}(A) \subseteq R .
$$

This ideal is $G_{l r}$-invariant and contains the determinantal ideal $I_{m}(A)$.

The annihilator-of-cokernel is a rather delicate invariant, for example it does not transform nicely under ring homomorphisms, ann.coker $(\phi(A)) \neq \phi($ ann.coker $(A))$. Yet, this ideal is controlled by the determinantal ideals as follows:

Property 3.3. (1) ann.coker $(A)^{m} \subseteq I_{m}(A) \subseteq \operatorname{ann.coker}(A) \subseteq \sqrt{I_{m}(A)}$,

and for any $m \geq j>1$ holds: $\operatorname{ann} . \operatorname{coker}(A) \cdot I_{j-1}(A) \subseteq I_{j}(A), \quad[$ Eisenbud, proposition 20.7].

(2) If $m=n$ and $\operatorname{det}(A)$ is not a zero divisor in $R$ then ann.coker $(A)=I_{m}(A): I_{m-1}(A)$.

(3) If $m<n$ and height $\left(I_{m}(A)\right)=(n-m+1)$ then ann.coker $(A)=I_{m}(A)$, [Eisenbud, exercise 20.6].

(4) If $I_{m-1}(A)$ contains a non-zero divisor modulo $I_{m}(A)$ then ann.coker $(A)=I_{m}(A)$, [Buchsbaum-Eisenbud, Corollary 1.4].

In particular, for one-row matrices, $m=1$, or when $I_{m}(A)$ is a radical ideal, $I_{m}(A)=\operatorname{ann} . \operatorname{coker}(A)$.

We use the following properties:

Lemma 3.4. (1) Block-diagonal case, ann.coker $(A \oplus B)=$ ann.coker $(A) \cap$ ann.coker $(B)$.

(2) For a square matrix, $m=n$, whose determinant is a non-zero divisor, holds: ann.coker $(A)=$ ann.coker $\left(A^{t}\right)$.

Suppose, moreover, either $R$ is a unique factorization domain (UFD) or height $\left(I_{m}(A)\right)<$ height $\left(I_{m-1}(A)\right)$, then ann.coker $(A)$ is a principal ideal.

(3) If $m>n$ then ann.coker $(A)=\{0\}$.

The statement 1 is immediate.

The first part of Statement $\mathbf{2}$ follows directly, e.g. from ann.coker $(A)=I_{m}(A): I_{m-1}(A)$ of property (3.3). For the second part of 2 note that for a square matrix $I_{m}(A)$ is a principal ideal and the height of ann.coker $(A) \subset R$ is one. If $R$ is UFD then ann.coker $(A)$ is generated by just one element.

Statement 3: in this case the submodule $\operatorname{Im}(A) \subset R^{\oplus m}$ is of $\operatorname{rank} \leq n<m$. Thus $\operatorname{rank}(\operatorname{coker}(A))>0$, i.e. this module has linearly independent elements, hence ann $(\operatorname{coker}(A))=\{0\}$. Geometrically, the module coker $(A)$ is supported on the whole $\operatorname{Spec}(R)$.

3.3. Skew-symmetric matrices and their Pfaffian ideals. Suppose $A$ is a square matrix then $I_{m}(A)=(\operatorname{det}(A))$. If moreover $A \in \operatorname{Mat}_{m \times m}^{\text {skew-sym }}(R)$, with $m$-even, then the determinant is a full square, $\operatorname{det}(A)=\operatorname{Pf}(A)^{2}$. Here the Pfaffian polynomial is explicitly written in terms of the entries of $A$, [Heymans], [Eisenbud, exercise A.2.11], [Greub]. In particular, the Pfaffian polynomial transforms under ring homomorphisms, $R \stackrel{\phi}{\rightarrow} S$ as $\operatorname{Pf}(\phi(A))=\phi(\operatorname{Pf}(A))$.

The transformation of the Pfaffian under the congruence is: $\operatorname{Pf}\left(U A U^{t}\right)=\operatorname{det}(U) \operatorname{Pf}(A)$.

If $m$ is odd then $\operatorname{det}(A)=0$. In this case, as a measure of degeneracy, we need the following refinement of the Pfaffian ideal:

$$
\left(\operatorname{Pf}_{m-1}(A)\right):=\sum_{i=1}^{m}\left(\operatorname{Pf}\left(A_{\widehat{i}}\right)\right) .
$$

Here $A_{\widehat{i}}$ is the $(m-1) \times(m-1)$ block of $A$, obtained by erasing the $i$ 'th row and column. 
We use the following properties of these ideals:

Lemma 3.5. Let $R$ be a ring of zero characteristic.

(1) Let $A \in M a t_{m \times m}^{\text {skew-sym }}(R)$ for m-even. There exists the "Pfaffian-adjoint" matrix, adj ${ }^{P f}(A) \in M a t_{m \times m}^{\text {skew-sym }}(R)$, that satisfies $A \cdot \operatorname{adj}^{P f}(A)=\operatorname{Pf}(A) \cdot \mathbb{I}=a d j^{P f}(A) \cdot A$. In particular, ann.coker $(A) \supseteq(P f(A))$.

(2) Moreover: $\operatorname{Span}_{R}\left(A U+U^{t} A\right)_{U \in M a t_{m \times m}(R)} \supseteq \operatorname{Mat}_{m \times m}^{\text {skew-sym }}(\operatorname{Pf}(A))$.

(3) Let $A \in M a t_{m \times m}^{\text {skew-sym }}(R)$ for $m>1$, odd. Then $\operatorname{Span}_{R}\left(A U+U^{t} A\right)_{U \in M_{a t} \times m(R)} \supseteq \operatorname{Mat}_{m \times m}^{\text {skew-sym }}\left(P f_{m-1}(A)\right)$.

Proof. 1. We recall the relevant exterior algebra, [Eisenbud, exercise A.2.11]. Let $V$ be a free $R$-module of rank $m$. Its dual, $V^{*}$, is the module of one-forms, take the dual basis $\left\{d x_{i}\right\}$. Associate to $A$ the skew-symmetric form $w_{A}:=\sum_{1 \leq i<j \leq m} a_{i j} d x_{i} \wedge d x_{j} \in \wedge^{2} V^{*}$. The top exterior power of $w_{A}$ gives the Pfaffian:

$$
\stackrel{\frac{m}{2}}{\wedge} w_{A}=m ! \cdot \operatorname{Pf}(A) d x_{1} \wedge \cdots \wedge d x_{m} \in \stackrel{m}{\wedge} V^{*} .
$$

Now, using the pairing $\stackrel{2}{\wedge} V^{*} \otimes \stackrel{m-2}{\wedge} V^{*} \rightarrow \stackrel{m}{\wedge} V^{*}$, we associate to the form $\frac{1}{m !} \stackrel{\frac{m}{2}-1}{\wedge} w_{A}$ an element of $\stackrel{2}{\wedge} V^{*}$, which we call $a d j j^{P f}(A)$. Then the relation $w_{A} \wedge\left(\stackrel{\frac{m}{2}-1}{\wedge} w_{A}\right)=\operatorname{Pf}(A) d x_{1} \wedge \cdots \wedge d x_{m}$ transforms into $A \cdot \operatorname{adj}{ }^{P f}(A)=\operatorname{Pf}(A) \cdot \mathbb{I}$.

2. This is now immediate, one takes $U$ in the form $\operatorname{adj}^{P f}(A) \cdot \tilde{U}$, where $\tilde{U} \in M a t_{m \times m}^{\text {skew-sym }}(R)$.

3. As in part 1 we have:

$$
\stackrel{\frac{m-1}{2}}{\wedge} w_{A}=(m-1) ! \sum_{i=1}^{m} \operatorname{Pf}\left(A_{\hat{i}}\right) d x_{1} \wedge \cdots \wedge \underbrace{\left(d x_{i}\right)}_{\text {omitted }} \wedge \cdots \wedge d x_{m} \in \stackrel{m-1}{\wedge} V^{*} .
$$

We get: $d x_{i} \wedge\left(\stackrel{\frac{m-3}{\wedge}}{\wedge} w_{A}\right) \wedge w_{A}=(m-1) ! P f\left(A_{\widehat{i}}\right) d x_{1} \wedge \cdots \wedge d x_{m}$.

Now, we use the pairing $\stackrel{2}{\wedge} V^{*} \otimes \stackrel{m-3}{\wedge} V^{*} \rightarrow \stackrel{m-1}{\wedge} V^{*}$, and the collection of forms $\left\{\left(\stackrel{\frac{m-3}{2}}{\wedge} w_{A}\right) \wedge d x_{i}\right\}_{i}$ to get:

$$
\operatorname{Span}_{R}\left(U_{i} A+A U_{i}^{t}\right)_{i} \supseteq\left(P f_{m-1}(A)\right) \cdot \stackrel{2}{\wedge} V^{*}=M a t_{m \times m}^{\text {skew-sym }}\left(P f_{m-1}(A)\right) .
$$

(Here the matrix $U_{i}$ corresponds to $\stackrel{\frac{m-1}{2}}{\wedge} w_{A} \wedge d x_{i}$.)

3.4. Integral closure of ideals. The integral closure of an ideal $I \subset R$ is defined as

$$
\bar{I}=\left\{f \in R \mid f^{d}+\sum_{j=1}^{d} a_{j} f^{d-j}=0, \text { for some } d \in \mathbb{N} \text { and some }\left\{a_{j} \in I^{j}\right\}_{j=1, \ldots, d}\right\} .
$$

This subset is an ideal in $R$ and is itself integrally closed, i.e. $\overline{\bar{I}}=\bar{I}$. We mention several useful properties:

Property 3.6. $\quad(1) I \subseteq \bar{I} \subseteq \sqrt{I} . \quad$ If $I \subset R$ is radical then $\bar{I}=I$.

If the height of $I \subset R$ is defined then height $(I)=\operatorname{height}(\bar{I})$;

(2) If $R$ is normal (i.e. integrally closed in its total ring of fractions) then every principal ideal is integrally closed, [Huneke-Swanson, Proposition 1.5.2].

(3) $\overline{I: J} \subseteq \bar{I}: J,[$ Huneke-Swanson, page 7].

(4) If $R$ is Noetherian then $\bar{I} \cdot \bar{J} \subseteq \overline{I \cdot J}$, [Huneke-Swanson, Corollary 6.8.6].

(5) If $R$ is a domain and $\{0\} \neq I$ is finitely generated then $\overline{I \cdot J}: \bar{I}=\bar{J}$, [Huneke-Swanson, Corollary 6.8.7].

(6) If $R$ is local, Noetherian and regular then $\overline{f \cdot J}=(f) \cdot \bar{J}$.

(7) Let $(R, \mathfrak{m})$ local Noetherian and $J \subset \mathfrak{m}$ an $\mathfrak{m}$-primary ideal. Then $\bar{I} \cdot \widehat{R}=\overline{I \cdot \widehat{R}}$, [Huneke-Swanson, Lemma 9.3.1].

In general the computation of $\bar{I}$ is algorithmically involved, it is realized in computer algebra packages as e.g. SINGULAR. The following criterion is highly useful in many cases, [Huneke-Swanson, Theorem 6.8.3]. For any ring homomorphism $R \stackrel{\phi}{\rightarrow} S$ denote by $S \phi(I)$ the ideal generated by the image of $I$ in $S$.

$$
\text { If } R \text { is Noetherian then } \bar{I}=\bigcap_{R \stackrel{\phi}{\rightarrow} S_{D V R}} \phi^{-1} S \phi(I)
$$

(Here the intersection goes over all the homomorphisms to discrete valuation rings.) In words:

$$
f \in \bar{I} \text { iff for any homomorphism to a DVR, } R \stackrel{\phi}{\rightarrow} S, \phi(f) \in S \phi(I) .
$$

This criterion has a simple geometric formulation (initially for $R=\mathbb{C}\{\underline{x}\}$, [Teissier, 1.3.4]):

$$
f \in \bar{I} \text { iff for any map of the smooth curve-germ, }(C, 0) \stackrel{\nu}{\rightarrow} \operatorname{Spec}(R) \text {, the pullbacks satisfy: } \nu^{*}(f) \in \nu^{*}(I) \text {. }
$$


3.5. Integral closure of modules. For the general definition of the integral closure of modules see [Huneke-Swanson, definition 16.1.1]. In our case all the modules are embedded into $R^{\oplus m}$, the ring is Noetherian over a field with $\operatorname{char}(\mathbb{k})=0$, thus the definition simplifies. For any ring homomorphism $R \stackrel{\phi}{\rightarrow} S$ take the image $\phi(M) \subseteq S^{\oplus m}$ and denote by $S \phi(M)$ the generated submodule.

Definition 3.7. [Huneke-Swanson, §16.1] The integral closure of a submodule $M \subset \mathbb{R}^{m}$ is $\bar{M}:=\bigcap_{R \rightarrow S_{D V R}} \quad \phi^{-1} S \phi(M)$.

In words: $z \in \bar{M}$ iff for any homomorphism to a discrete valuation domain, $R \stackrel{\phi}{\rightarrow} S_{D V R}$, there holds $\phi(z) \in S \phi(M)$.

For any ideals $J_{1}, J_{2} \subset R$ holds, [Huneke-Swanson, Remark 1.3.2]:

$$
J_{1} \cdot J_{2} \subseteq J_{1} \cdot \overline{J_{2}} \subseteq \overline{J_{1}} \cdot \overline{J_{2}} \subseteq \overline{J_{1} \cdot J_{2}} .
$$

More generally:

Lemma 3.8. Let $R$ be Noetherian, $N \subseteq M \subseteq R^{\oplus m}$ embedded modules, $J \subset R$ an ideal.

(1) $J \cdot \bar{M} \subseteq \bar{J} \cdot \bar{M} \subseteq \bar{J} \cdot \bar{M}$.

(2) $\operatorname{ann} \bar{M} / \mathrm{N} \supseteq \overline{\operatorname{ann} M / N}$.

Proof. 1. The first inclusion is trivial. For the second it is enough to prove: for any $f \in \bar{J}, z \in \bar{M}$ holds $f \cdot z \in \bar{J} \cdot M$. Let $R \stackrel{\phi}{\rightarrow} S_{D V R}$ then $\phi(f) \in S \cdot \phi(J)$ and $\phi(z) \in S \cdot \phi(M)$. Thus $\phi(f z)=\phi(f) \phi(z) \in S \cdot \phi(J) \cdot \phi(M)=S \cdot \phi(J \cdot M)$. As this holds for any such $\phi$ we get $f \cdot z \in \overline{J \cdot M}$.

2. Let $f \in \overline{a n n} \frac{M / N}{N}$, i.e. for any $R \stackrel{\phi}{\rightarrow} S_{D V R}$ holds $\phi(f) \in \phi(a n n M / N)$. Thus $f \in a n n M / N+k e r(\phi)$, i.e. $f \cdot M \subseteq N+k e r(\phi)$. But then $\phi(f) \phi(M) \subseteq \phi(N)$. As this holds for any $\phi$, we get: $\overline{f \cdot M} \subseteq \bar{N}$. Finally, by part (1.), $f \cdot \bar{M} \subseteq \overline{f \cdot M} \subseteq \bar{N}$, i.e. $f \in$ ann $\bar{M} / \bar{N}$.

Example 3.9. The ideals in part (2) of Lemma 3.8 can differ significantly. For example, let $R=\mathbb{k}\left[\left[x_{1}, \ldots, x_{p}\right]\right]$, with $p \geq 3$, and let $N=\left(x_{1}^{d}, \ldots, x_{p}^{d}\right) \subsetneq M=\mathfrak{m}^{d}$. Then $\bar{N}=\bar{M}=\mathfrak{m}^{d}$, hence $a n n \bar{M} / N=R$. But $\overline{a n n} \frac{M / N}{N}=\mathfrak{m}^{d}$.

Proof: obviously $N \subseteq$ ann $M / N$, thus $\overline{\text { ann } M / N} \supseteq \bar{N}=\mathfrak{m}^{d}$. Furthermore, $\overline{\text { ann } M / N}$ is a monomial ideal. Suppose $i_{1}+\cdots+i_{p}=d-1$ and $x_{1}^{i_{1}} \cdots x_{p}^{i_{p}} \in \overline{\text { ann } M / N} \backslash \mathfrak{m}^{d}$. Take the monomial $x^{d-1-i_{1}} \cdots x_{p}^{d-1-i_{p}} \in \mathfrak{m}^{p(d-1)-(d-1)} \subset \mathfrak{m}^{d}=M$. Then

$$
\left(x^{d-1-i_{1}} \cdots x_{p}^{d-1-i_{p}}\right) \cdot\left(x_{1}^{i_{1}} \cdots x_{p}^{i_{p}}\right)=x_{1}^{d-1} \cdots x_{p}^{d-1} \notin N .
$$

Hence $\left(x_{1}^{i_{1}} \cdots x_{p}^{i_{p}}\right) \cdot M \nsubseteq N$, contradicting the assumption $x_{1}^{i_{1}} \cdots x_{p}^{i_{p}} \in \overline{\operatorname{ann} M / N}$.

The following lemma compares the bounds in part three of Theorem 2.2.

Lemma 3.10. If $R$ is a Noetherian ring then $\overline{\text { ann.coker }(A)} \subseteq \overline{I_{m}(A)}: \overline{I_{m-1}(A)}$.

Indeed, by Property (3.3) we have ann.coker $(A) \cdot I_{m-1}(A) \subseteq I_{m}(A)$. Thus the statement follows from part 1 of Lemma 3.8 .

3.6. The largest "reasonable" group is $G_{l r}$ (matrix preservers over rings). When looking for the possible groups acting on matrices, $G \circlearrowright \operatorname{Mat}_{m \times n}(R)$, the guiding principle is that the action of $G$ should, at least, distinguish between degenerate and non-degenerate matrices. Groups with such properties are restricted by the following proposition. For the general introduction to the theory of preservers, i.e. self-maps of $\operatorname{Mat}_{m \times n}(\mathbb{k})$, that preserve some properties/structures see [Molnár], [Li-Pierce].

Proposition 3.11. Let $R$ be a unique factorization domain with at least four elements. Let $T$ be an invertible map acting $R$-linearly on the module $\operatorname{Mat}_{m \times n}(R)$, for $m \leq n$, possibly violating the row/column structure. Suppose $T$ preserves the set of degenerate matrices, i.e. $I_{m}(A)=\{0\}$ iff $I_{m}(T(A))=\{0\}$. Then either $T(A)=U A V$, for some $U \in G L(m, R), V \in G L(n, R)$, or, when $m=n, T(A)=U A^{t} V$.

Proof. We pass to the field of fractions $\operatorname{Frac}(R)$. As $R$ has no zero-divisors, it is naturally embedded, $R \subset F r a c(R)$. Thus $T$ extends to an invertible operator that acts $\operatorname{Frac}(R)$-linearly on the vector space of matrices $\operatorname{Mat} t_{m \times n}(\operatorname{Frac}(R))$ and such that $I_{m}(A)=\{0\}$ if and only if $I_{m}(T(A))=\{0\}$. In other words, $T$ preserves the degenerate matrices, of $r a n k<m$, and also preserves the non-degenerate matrices of rank $=m$. Now we use the classical results on the preservers over a field:

i. (the case $m=n$ ) [Dieudonné, Theorem 3]: Let $\mathbb{k}$ be an arbitrary field. Any invertible $\mathbb{k}$-linear transformation of $M a t_{m \times m}(\mathbb{k})$ that preserves the set of degenerate matrices has either the form $T(A)=U A V$ or the form $T(A)=U A^{t} V$, for some $U, V \in G L(m, \mathbb{k})$.

ii. (the case $m<n$ ) [Beasley-Laffey, Theorem 7] Suppose the field $\mathbb{k}$ contains at least four elements. Any invertible $\mathbb{k}$-linear transformation of $\operatorname{Mat}_{m \times n}(\mathbb{k})$ that preserves the set of rank $=r$ matrices (for some fixed $0<r \leq m$ ) is of the form $T(A)=U A V$ for some $U \in G L(m, \mathbb{k}), V \in G L(n, \mathbb{k})$.

Therefore we get: either $T(A)=U A V$, for some $U \in G L(m, F r a c(R)), V \in G L(m, F r a c(R))$, or, for $m=n, T(A)=U A^{t} V$. It remains to show that we can choose $U \in G L(m, R), V \in G L(n, R)$. (We check the case $m<n$, the case $m=n$ is similar.)

Let $0 \neq g \in R$ be a(ny) common denominator of the entries of $U$, i.e. $g \cdot U \in M a t_{m \times n}(R)$. Replace $(U, V)$ by $\left(g \cdot U, \frac{V}{g}\right)$, so we still have $T(A)=U A V$.

Substitute for $A$ the elementary matrix $E_{i j}$, with 1 at the i'th row, j'th column and zeros otherwise. The $p, q$-entry of the matrix $U E_{i j} V$ equals $u_{p i} v_{j q}$ and it belongs to $R$. Suppose some element of $V$ is not in $R$, we can assume it is $v_{11}$. Present $v_{11}$ as a fraction, $v_{11}=\frac{f}{g}$, for some $f, g \in R$. As $R$ is UFD we can assume that $f, g$ are co-prime, i.e. $(f) \cap(g)=(f g)$ and $g$ is not invertible in $R$. 
As $u_{p i} v_{11} \in R$, for any $p, i$, we get: $U$ is divisible by $g$. Thus we pass from $(U, V)$ to $\left(\frac{U}{g}, g V\right)$ and proceed to the other entries of $V$.

Therefore, in our work the group $G \circlearrowright M a t_{m \times n}(R)$ is always a subgroup of $G_{l r}=G L_{R}(m) \times G L_{R}(n)$.

Remark 3.12. The last statement is very sensitive to the particular form of condition " $T$ preserves degenerate matrices". For example, when the ring is local, one could ask for a condition: $T(A) \in \operatorname{Mat}_{m \times n}\left(\mathfrak{m}^{q}\right)$ iff $A \in M a t_{m \times n}\left(\mathfrak{m}^{q}\right)$. But this condition is satisfied just by $R$-linearity of $T \circlearrowright \operatorname{Mat}_{m \times n}(R)$, without any additional restrictions.

3.7. Unipotent subgroups. Fix a group action on an $R$-module $G \circlearrowright M$ and a decreasing filtration $M=M_{0} \supsetneq M_{1} \supsetneq \cdots$. We get the induced filtration of $G$ by the (normal) subgroups

$$
G^{(i)}:=\left\{g \in G \mid \forall j \geq 0 \quad \forall z_{j} \in M_{j}: g\left(z_{j}\right)-z_{j} \in M_{j+i}\right\} .
$$

In particular, the subgroup $G^{(0)} \subseteq G$ preserves the filtration, while the subgroup $G^{(1)} \subseteq G$ acts unipotently. We call $G^{(1)}$ the "unipotent" subgroup of $G$. If $G^{(\overline{1})}=G$ then $G$ itself is called "unipotent for the given filtration". The general properties of the filtration $G \supseteq G^{(0)} \supseteq G^{(1)} \supseteq \cdots$ are studied in [B.K.1, §3].

For the goals of the current paper we usually need the filtration by the powers of a/the maximal ideal,

$$
\operatorname{Mat}_{m \times n}(R) \supsetneq M_{m \times n}(\mathfrak{m}) \supsetneq \operatorname{Mat}_{m \times n}\left(\mathfrak{m}^{2}\right) \cdots .
$$

Therefore we denote the unipotent subgroup by $G^{(\mathfrak{m})} \subseteq G$. In particular, when $R$ is a local ring:

$$
G_{r}^{(\mathfrak{m})}=\left\{U=\mathbb{I}+\tilde{U} \mid \tilde{U} \in M a t_{n \times n}(\mathfrak{m})\right\}, \quad G_{l}^{(\mathfrak{m})}=\left\{U=\mathbb{I}+\tilde{U} \mid \tilde{U} \in M a t_{m \times m}(\mathfrak{m})\right\}, \quad G_{l r}^{(\mathfrak{m})}=G_{l}^{(\mathfrak{m})} \times G_{r}^{(\mathfrak{m})} .
$$

The last equality is a statement, but the proof is straightforward, the conditions $\left\{U E_{i j} V-E_{i j} \in M a t_{m \times n}(\mathfrak{m})\right\}_{i, j}$ force $U \in G_{l}^{(\mathfrak{m})}$, $V \in G_{r}^{(\mathfrak{m})}$.

More generally, for any subgroup $G \subset G_{l r}$ we have: $G^{(m)}=G \cap G_{l r}^{(\mathfrak{m})}$.

3.8. The tangent spaces. The tangent spaces to the groups and group-orbits are defined and studied in great generality in [B.K.1, §3.7]. Here we recall them for our group actions.

We use the isomorphism of $R$-modules, $T_{\left(M_{m \times n}(R), A\right)} \stackrel{\sim}{\longrightarrow} M a t_{m \times n}(R)$, to identify $T_{(G A, A)}$, $T_{(\Sigma, A)}$ with their images in $\operatorname{Mat}_{m \times n}(R)$.

Example 3.13. (See [B.K.1, §3.8].)

i. $\Sigma=\operatorname{Mat}_{m \times n}(R)$ and $G_{l r}: A \rightarrow U A V^{-1}$. Here $T_{\left(G_{l r} A, A\right)}=\operatorname{Span}_{R}\{u A, A v\}_{(u, v) \in M_{a t} \times m}(R) \times M a t_{n \times n}(R)$. Similarly for $G_{l}$ and $G_{r}$.

ii. $\Sigma=M_{a t}^{\text {sym }}(R)$ or $M a t_{m \times m}^{\text {skew-sym }}(R)$ and $G_{\text {congr }}: A \rightarrow U A U^{t}$. Here $T_{\left(G_{\text {congr }} A, A\right)}=\operatorname{Span}_{R}\left\{u A+A u^{t}\right\}_{u \in M a t_{m \times m}(R)}$.

iii. $\Sigma=\operatorname{Mat}_{m \times m}(R)$ and $G_{c o n j}: A \rightarrow U A U^{-1}$. Here $T_{\left(G_{c o n j} A, A\right)}=\operatorname{Span}_{R}\{u A-A u\}_{u \in M a t_{m \times m}(R)}$.

iv. $\Sigma=M_{\left.a t_{i}\right\} \times\left\{n_{j}\right\}}^{u p}(R)$ and $G_{l r}^{u p}: A \rightarrow U A V$, where $U, V$ are upper-block-triangular. Here

$$
T_{\left(G_{l r} A, A\right)}=\operatorname{Span}_{R}\{u A, A v\}_{(u, v) \in M a t_{\left\{m_{i}\right\} \times\left\{m_{j}\right\}}^{u p}(R) \times M a t_{\left\{n_{i}\right\} \times\left\{n_{j}\right\}}^{u p}(R)} \cdot
$$

Similarly for $G_{r}^{u p}$.

In all these cases holds: $T_{\left(G^{(\mathfrak{m})} A, A\right)}=\mathfrak{m} \cdot T_{(G A, A)}$. In general, for all the "reasonable" group actions there holds

$$
\left.\mathfrak{m} \cdot T_{(G A, A)} \subseteq T_{(G(\mathfrak{m})} A, A\right) \subseteq T_{(G A, A)},
$$

see [B.K.1, §3.4] for more detail.

3.9. Transition to the completion. Fix some proper ideal $\{0\} \neq \mathfrak{a} \subsetneq R$. Here $\mathfrak{a}$ is not necessarily a maximal ideal, as we need these results for partial algebraization, $\S 2.7$, and relative determinacy, $\S 2.8$. We often take the a-adic completion, $R \stackrel{\hat{\psi}}{\rightarrow} \widehat{R}$, and compute over $\widehat{R}$. To translate the results to $R$ we need the following standard facts.

Given two embedded modules, $M_{1} \subset M_{2} \subset R^{\oplus p}$, take their images under the completion map, $\hat{\psi}\left(M_{1}\right) \subseteq \hat{\psi}\left(M_{2}\right) \subseteq \hat{\psi}(R)^{\oplus p}$. These are not necessarily $\widehat{R}$-modules (unless the completion map is surjective), but they generate the $\widehat{R}$-modules: $\widehat{R} \cdot \hat{\psi}\left(M_{1}\right) \subseteq$ $\widehat{R} \cdot \hat{\psi}\left(M_{2}\right) \subseteq \widehat{R}^{\oplus p}$.

Note that in general $\hat{\psi}\left(M_{i}\right), \widehat{R} \cdot \hat{\psi}\left(M_{i}\right)$ differ from the "non-embedded" completion, $\widehat{M}=\underset{\leftarrow}{\lim / \mathfrak{a}^{n} \cdot M}$. (For example, fix a non-zero divisor $f \in \mathfrak{a}^{\infty}$ and consider $J=(f)$. Then $\hat{\psi}(J)=\{0\} \subset \widehat{R}$, while $\widehat{J} \approx \widehat{R}$, as $\widehat{R}$-modules.

In the statements below we use the Jacobson ideal of the ring, $J(R)$. Recall, for a local ring $J(R)=\mathfrak{m}$.

First we address the behaviour of the Loewy length of an ideal, $l l_{R}(J)=\min \left\{n \mid J \supseteq \mathfrak{a}^{n}\right\}$, under completion.

Lemma 3.14. Fix an ideal in a commutative unital ring, $\{0\} \neq \mathfrak{a} \subsetneq R$. Fix some other ideal, $\mathfrak{a}^{\infty} \subseteq J \subset R$. Suppose:

i. either the completion map is surjective, $R \stackrel{\hat{\psi}}{\rightarrow} \widehat{R}$;

ii. or both $\mathfrak{a}$ and $J$ are finitely generated (as $R$-modules) and $\mathfrak{a} \subseteq J(R)$.

Then $J \supseteq \mathfrak{a}^{n}$ iff $\widehat{R} \cdot \hat{\psi}(J) \supseteq \widehat{R} \cdot \hat{\psi}(\mathfrak{a})^{n}$. Equivalently: $l_{R}(J)=l l_{\widehat{R}}(\widehat{R} \cdot \hat{\psi}(J))$.

Proof. The part $\Rightarrow$ is immediate, as the ideal $\widehat{R} \cdot \hat{\psi}(\mathfrak{a})^{n}$ is generated by the images of generators of $\mathfrak{a}^{n}$.

$\Leftarrow$ Suppose $R \stackrel{\hat{\psi}}{\rightarrow} \widehat{R}$, then $\widehat{R} \cdot \hat{\psi}(J)=\hat{\psi}(J)$. If $\hat{\psi}(J) \supseteq \hat{\psi}(\mathfrak{a})^{n}$, then $J+\mathfrak{a}^{\infty} \supseteq \mathfrak{a}^{n}$. (By taking some preimages of the elements of $\hat{\psi}(\mathfrak{a})^{n}$ in $J$.) Thus, as $J \supseteq \mathfrak{a}^{\infty}$, we get $J \supseteq \mathfrak{a}^{n}$.

Suppose $\mathfrak{a}, J$ are finitely generated, then the inclusion follows by the standard Nakayama arguments. The completion morphism induces $R / \mathfrak{a}^{N} \sim \widehat{R} \widehat{R} \cdot \hat{\psi}(\mathfrak{a})^{N}$, for any $N$, and similarly $J+\mathfrak{a}^{N} / \mathfrak{a}^{N} \stackrel{\sim}{\longrightarrow} \cdot \hat{\psi}(J)+\widehat{R} \cdot \hat{\psi}(\mathfrak{a})^{N} \widehat{R} \cdot \hat{\psi}(a)^{N} \cdot$ Thus $\widehat{R} \cdot \hat{\psi}(J) \supseteq \widehat{R} \cdot \hat{\psi}(\mathfrak{a})^{n}$ 
implies $J+\mathfrak{a}^{N} / \mathfrak{a}^{N} \supseteq \mathfrak{a}^{n} / \mathfrak{a}^{N}$, or, equivalently, $J+\mathfrak{a}^{N} \supseteq \mathfrak{a}^{n}$, for any $N$. Therefore, for the embedding of (finitely generated) modules $J \subseteq J+\mathfrak{a}^{n}$, we have $J+\mathfrak{a}\left(J+\mathfrak{a}^{n}\right) \supseteq J+\mathfrak{a}^{n}$. And by Nakayama we have $J=J+\mathfrak{a}^{n}$, hence $J \supseteq \mathfrak{a}^{n}$.

Now we check the change of the annihilator of the quotient, ann $M_{2} / M_{1}$, under the completion.

Lemma 3.15. Fix an ideal in a commutative unital ring, $\{0\} \neq \mathfrak{a} \subsetneq R$, and two modules, $M_{1} \subset M_{2} \subseteq R^{\oplus p}$, with $M_{1} \supseteq$ $M_{2} \cap\left(\mathfrak{a}^{\infty} \cdot R^{\oplus p}\right)$. Suppose:

$i$. either the completion map is surjective, $R \stackrel{\hat{\psi}}{\rightarrow} \widehat{R}$;

ii. or $R$ is Noetherian and $M_{2}$ is a finitely generated $R$-module and $\mathfrak{a} \subseteq J(R)$.

Then ann $M_{2} / M_{1} \supseteq \mathfrak{a}^{n}$ iff ann $\left(\widehat{R} \cdot \hat{\psi}\left(M_{2}\right) / \widehat{R} \cdot \hat{\psi}\left(M_{1}\right)\right) \supseteq \widehat{R} \cdot \hat{\psi}\left(\mathfrak{a}^{n}\right)$. Equivalently: ll $l_{R}\left(\right.$ ann $\left.M_{2} M_{1}\right)=l l_{\widehat{R}}\left(\right.$ ann $\left.\widehat{R} \cdot \hat{\psi}\left(M_{2}\right) \widehat{\widehat{R}} \cdot \hat{\psi}\left(M_{1}\right)\right)$.

Proof. $\Rightarrow$ Direct check: $\widehat{R} \cdot \hat{\psi}\left(\mathfrak{a}^{n}\right) \cdot \widehat{R} \cdot \hat{\psi}\left(M_{2}\right)=\widehat{R} \cdot \hat{\psi}\left(\mathfrak{a}^{n} \cdot M_{2}\right) \subseteq \widehat{R} \cdot \hat{\psi}\left(M_{1}\right)$.

$\Leftarrow$ Suppose $\widehat{R} \cdot \hat{\psi}\left(\mathfrak{a}^{n}\right) \cdot \widehat{R} \cdot \hat{\psi}\left(M_{2}\right) \subseteq \widehat{R} \cdot \hat{\psi}\left(\mathfrak{a}^{n} \cdot M_{1}\right)$.

In the case of surjective completion, $R \stackrel{\hat{\psi}}{\rightarrow} \widehat{R}$, we get $\mathfrak{a}^{n} M_{2} \subseteq M_{1}+\mathfrak{a}^{\infty} R^{\oplus p}$, by choosing some preimages under $\hat{\psi}$. Then, by direct check, $\mathfrak{a}^{n} M_{2} \subseteq M_{1}+M_{2} \cap \mathfrak{a}^{\infty} R^{\oplus p}=M_{1}$.

Otherwise, when $R$ is Noetherian, $\mathfrak{a}, M_{2}$ are finitely generated, and we use the Nakayama argument. As in the previous lemma, we have: $\mathfrak{a}^{n} M_{2} \subseteq M_{1}+\left(M_{2} \cap \mathfrak{a}^{N} R^{\oplus p}\right)$, for any $N$. By Artin-Rees lemma, for $N \gg 1$ we have: $\left(M_{2} \cap \mathfrak{a}^{N} R^{\oplus p}\right) \subseteq \mathfrak{a}^{n+1} M_{2}$. Thus we have $\mathfrak{a}^{n} M_{2} \subseteq M_{1}+\mathfrak{a}^{n+1} M_{2}$. Now the Nakayama lemma gives $\mathfrak{a}^{n} M_{2} \subseteq M_{1}$.

Lemma 3.16. As before, fix two modules, $M_{1} \subset M_{2} \subseteq R^{\oplus p}$, with $M_{1} \supseteq M_{2} \cap\left(\mathfrak{a}^{\infty} \cdot R^{\oplus p}\right)$. Suppose the a-adic completion is surjective, $R \stackrel{\hat{\psi}}{\rightarrow} \widehat{R}$, then $\hat{\psi}\left(\right.$ ann $\left.M_{2} / M_{1}\right)=\operatorname{ann} \hat{\psi}\left(M_{2}\right) / \hat{\psi}\left(M_{1}\right)$.

Proof. The part $\subseteq$ is straightforward: $\hat{\psi}\left(\right.$ ann $\left.M_{2} / M_{1}\right) \cdot \hat{\psi}\left(M_{2}\right)=\hat{\psi}\left(\operatorname{ann}\left(M_{2} / M_{1}\right) \cdot M_{2}\right) \subseteq \hat{\psi}\left(M_{1}\right)$.

The part $\supseteq$. By the surjectivity $R \rightarrow \widehat{R}$ any element of ann $\hat{\psi}\left(M_{2}\right) \hat{\psi}\left(M_{1}\right)$ is of the form $\hat{\psi}(f)$ for some $f \in R$. As $\hat{\psi}(f) \hat{\psi}\left(M_{2}\right) \subseteq$ $\hat{\psi}\left(M_{1}\right)$ we get: $f M_{2} \subseteq M_{1}+\left(M_{2} \cap \mathfrak{a}^{\infty} R^{\oplus p}\right)=M_{1}$. Thus $f \in$ ann $M_{2} / M_{1}$.

Corollary 3.17. Fix two ideals, $I, J \subset R$, with $I \supseteq\left((I+J) \cap \mathfrak{a}^{\infty}\right)$.

(1) Suppose the completion is surjective, $R \rightarrow \widehat{R}$, then $\hat{\psi}(I: J)=\hat{\psi}(I): \hat{\psi}(J)$.

(2) Suppose $R$ is Noetherian and $\mathfrak{a} \subseteq J(R)$, then $l_{R}(I: J)=l l_{\widehat{R}}(\widehat{R} \hat{\psi}(I): \widehat{R} \hat{\psi}(j))$.

(3) Suppose $(R, \mathfrak{m})$ is a local Noetherian ring and $A \in \operatorname{Mat}_{m \times n}(R)$ satisfies: $I_{m}(A)$ is an $\mathfrak{m}-$ primary ideal. Then, for the $\mathfrak{m}$-completion $R \stackrel{\hat{\psi}}{\rightarrow} \widehat{R}$ holds: $l l_{R}\left(\overline{I_{m}(A)}: \overline{I_{m-1}(A)}\right)=l l_{\widehat{R}}\left(\overline{I_{m}(\widehat{A})}: \overline{I_{m-1}(\widehat{A})}\right)$.

Proof. Note that $I: J=a n n I+J / I$.

1. Apply part one of Lemma 3.16 to the modules $I \subseteq I+J \subset R$. We get: $\hat{\psi}($ ann $I+J / I)=$ ann $(\hat{\psi}(I+J) / \hat{\psi}(I))$, hence the statement.

2. Follows by Lemma 3.15 .

3. By part 1 we have: $\left.l l_{R}\left(\overline{I_{m}(A)}: \overline{I_{m-1}(A)}\right)=l_{\widehat{R}}\left(\overline{\left(I_{m}(A)\right.} \cdot \widehat{R}\right):\left(\overline{I_{m-1}(A)} \cdot \widehat{R}\right)\right)$. By the integral closure properties, Property 3.6, we have: $\overline{I_{j}(A)} \cdot \widehat{R}=\overline{I_{j}(A) \cdot \widehat{R}}$. Finally, $I_{j}(A) \cdot \widehat{R}=I_{j}(A \cdot \widehat{R})=I_{j}(\widehat{A})$.

3.10. The "relevant approximation" property. To establish finite determinacy we should resolve the condition $g z=z+w$, $g \in G$. In most cases this is a (finite) system of equations. First one looks for an order-by-order solution (modulo the powers of the maximal ideal). Then one tries to establish an ordinary solution for these equations.

For the criteria in the Subsection 3.11 we need the "relevant approximation property of the ring $R$ ". The particular property depends on the type of the equations. While the general theory is developed in [B.K.1], for the subgroups of $G_{l r}$ we need only the following two types of approximation property.

3.10.1. If $G \subseteq G_{l r}$ is defined by $R$-linear equations and the condition $g A=A+B$ can be written as a system of linear equations on $g=(U, V)$ then we say that $R$ has the relevant approximation property if the assumption (3) together with the condition ann.coker $(A) \supseteq \mathfrak{m}^{\infty}$ hold.

The equations are linear for the groups $G_{r}, G_{l}, G_{l r}, C_{c o n j}$. They are:

$$
U A=A+B, \quad A V=A+B, \quad U A=(A+B) V, \quad U A=(A+B) U .
$$

The class of rings satisfying (3) is rather large. Besides the traditional Noetherian rings of Commutative Algebra this class contains e.g. the ring $C^{\infty}\left(\mathbb{R}^{p}, 0\right)$ of infinitely differentiable function-germs in several variables. Its completion is Noetherian, $\mathbb{R} \llbracket \underline{x} \rrbracket$, and the completion map, $C^{\infty}\left(\mathbb{R}^{p}, 0\right) \rightarrow \mathbb{R} \llbracket \underline{x} \rrbracket$, is surjective by Borel's lemma, [Rudin, pg. 284, exercise 12].

For the non-Noetherian rings, with $\mathfrak{m}^{\infty} \neq\{0\}$ the condition ann.coker $(A) \supseteq \mathfrak{m}^{\infty}$ can be checked using the classical Łojasiewicz property:

If $f \in C^{\infty}\left(\mathbb{R}^{p}, 0\right)$ satisfies $|f| \geq C|\underline{x}|^{\delta}$, for some $C>0, \delta>0$,

then $f$ divides any function flat at the origin, and thus $(f) \supseteq \mathfrak{m}^{\infty}$. 
3.10.2. If $G \subseteq G_{l r}$ is defined by polynomial/analytic equations and the condition $g A=A+B$ can be written as a system of polynomial/analytic equations on $g=(U, V)$, we say that $R$ has the relevant approximation property provided $R$ is Henselian ring. This is the case for $G_{\text {congr }}$, the equations being quadratic, $U A U^{t}=A+B$.

3.11. The transition from $\operatorname{ann}\left(T_{(\Sigma, G, A)}^{1}\right)$ to the finite determinacy and the main results of [B.K.1]. In this section $(R, \mathfrak{m})$ is a local ring over a field, $\mathbb{k}$, of zero characteristic.

Fix a finitely generated $R$-module with descending filtration, $M=M_{0} \supset M_{1} \supset \cdots$. We assume that the filtration is "essentially descending", i.e. for any $j$ there exists $k_{j}$ such that $M_{k_{j}} \subseteq \mathfrak{m}^{j} \cdot M$. Fix a (filtered) group action, $G \circlearrowright M$, and a deformation space $\Sigma \subseteq M$. (In our case $M=\operatorname{Mat}_{m \times n}(R)$ or $\operatorname{Mat}_{m \times m}^{\text {sym }}(R)$ or $M a t_{m \times m}^{\text {skew-sym }}(R)$, and $\Sigma \subseteq M$ is a submodule.) To understand the "essential" deformations of an element $z \in \Sigma$, we need to know "how small/large" is the orbit $G z$ as compared to $\Sigma$. In many cases this question reduces to the linear version:

What is the biggest $R$-submodule $\Lambda \subset M$ satisfying: $z+\Lambda \subseteq G z$ ?

(The precise statement is [B.K.1, Lemma 2.1].)

The main result of [B.K.1] is the transition of this linear version to the comparison of the tangent spaces, $T_{(G z, z)} \subseteq T_{(M, z)}$. We assume that the germ $(G z, z)$ is "nice", in particular it possesses a well defined tangent space. The precise condition is:

the subgroup $G \subset G L_{\mathbb{k}}(M)$ and its completion $\widehat{G} \subseteq G L_{\mathbb{k}}(\hat{M})$ are $\mathbb{k}$-polynomially-defined; their unipotent parts, $G^{(1)}, \widehat{G}^{(1)}$, are of Lie type.

The condition " $G$ is of Lie type" ensures that the tangent space $T_{(G z, z)}$ "approximates" the germ $(G z, z)$. These properties hold for all our groups, $G_{l}, G_{r}, G_{l r}, G_{c o n g r}, G_{c o n j}, \ldots$, see the details in [B.K.1, §3.7].

Furthermore, we use the isomorphism of $R$-modules $T_{(M, z)} \stackrel{\sim}{\longrightarrow}$ to identify $T_{(G z, z)}$ with its embedding. Accordingly, we have the embedded module: $T_{(G z, z)} \subseteq M$, as in $\S 3.8$.

Theorem 3.18. [B.K.1, Theorem 2.2] Suppose the (filtered) action $G \circlearrowright\left\{M_{i}\right\}$ satisfies assumptions (28). Suppose that G is unipotent for the filtration $\left\{M_{i}\right\}$.

(1) If $M_{i} \subseteq T_{(G z, z)}$ and $R$ has the relevant approximation property (§3.10) then $\{z\}+M_{i} \subseteq G z$.

(2) Suppose $T_{(G z, z)} \subseteq T_{(M, z)}$ is an $R$-submodule. If $\{z\}+M_{i} \subseteq G z$ then $M_{i} \subseteq T_{(G z, z)}$.

While Theorem 3.18 is quite general, to compute/bound the order of determinacy we use a more specific criterion (see $\S 2.1$ for the Loewy length $\left.l l_{R}(.).\right)$ :

Proposition 3.19. Suppose $\Sigma \subseteq \operatorname{Mat}_{m \times n}(R)$ is a free direct summand, i.e. $\Sigma \oplus \Sigma^{\perp}=$ Mat $t_{m \times n}(R)$ for a free submodule $\Sigma^{\perp} \subset \operatorname{Mat}_{m \times n}(R)$.

(1) [B.K.1, Corollary 2.6] Suppose ann $\left(T_{(\Sigma, G, A)}^{1}\right) \supseteq \mathfrak{m}^{\infty}$ and $R$ has the relevant approximation property. Then

$$
l l_{R}\left(\operatorname{ann}\left(T_{(\Sigma, G, A)}^{1}\right)\right)-1 \leq \operatorname{ord}_{G}^{\Sigma}(A) \leq l l_{R}\left(\operatorname{ann}\left(T_{\left(\Sigma, G^{(\mathfrak{m})}, A\right)}^{1}\right)\right)-1 .
$$

(2) If $\operatorname{ann}\left(T_{(\Sigma, G, A)}^{1}\right) \nsupseteq \mathfrak{m}^{\infty}$, then $A$ is not infinitely- $(\Sigma, G)$-determined.

For all our groups holds

thus we have the bounds on the annihilator:

$$
\mathfrak{m} \cdot \operatorname{ann}\left(T_{(\Sigma, G, A)}^{1}\right) \subseteq \operatorname{ann}\left(T_{\left(\Sigma, G^{(\mathfrak{m})}, A\right)}^{1}\right) \subseteq \operatorname{ann}\left(T_{(\Sigma, G, A)}^{1}\right) .
$$

So, the bounds in the corollary differ at most by one. Moreover, in many cases $\operatorname{ann}\left(T_{(\Sigma, G, A)}^{1}\right)=\operatorname{ann}\left(T_{(\Sigma, G(\mathfrak{m}), A)}^{1}\right)$, see $\S 3.8$.

This proposition reduces the determinacy question to the study of $T_{(\Sigma, G, A)}^{1}$ and its annihilator.

Remark 3.20. We mention the relation to the Castelnuovo-Mumford regularity of a module. Recall, [Eisenbud, exercise 20.18]: if $M=\oplus_{i} M_{i}$ is a graded $R$-module of finite length then its regularity equals $\operatorname{reg}(M)=\max \left\{i \mid M_{i} \neq\{0\}\right\}$. Therefore, when $T_{(\Sigma, G, A)}^{1}$ is graded and of finite length, we can rewrite:

$$
\operatorname{reg}\left(T_{(\Sigma, G, A)}^{1}\right) \leq \operatorname{ord}_{G}^{\Sigma}(A) \leq \operatorname{reg}\left(T_{\left(\Sigma, G^{(\mathfrak{m})}, A\right)}^{1}\right)
$$

3.12. Finite determinacy vs stable algebraizability. Let $\mathbb{k}[\underline{x}] I \subseteq R \subsetneq \widehat{R}=\mathbb{k}[[\underline{x}] / \mathbb{k}[[\underline{x}]]$. I be a ring over the field $\mathbb{k}$ of zero characteristic. The typical examples of $R$ are: $\mathbb{k}[\underline{x}] /, \mathbb{k}\langle\underline{x}\rangle / I$ (algebraic power series), $\mathbb{k}\{\underline{x}\} / I$ (convergent power series). In this section we do not use any matrix structure, thus instead of the modules $\operatorname{Mat}_{m \times n}(R), \operatorname{Mat}_{m \times n}(\widehat{R})$ we work with just free modules $F \subset \hat{F}:=\widehat{R} \cdot F$. By the assumption on $R$ the completion is injective, thus $F \subset \hat{F}$.

Fix a group action $G \circlearrowright \hat{F}$, $\mathbb{k}$-linear but not necessarily $\widehat{R}$-linear. We assume $G$ to be complete with respect to the filtration $\left\{G^{(i)}\right\}$ and moreover of Lie-type (see $\left.\S 3.11\right)$. Then the unipotent part $G^{(1)}$ admits the surjective exponential map, $T_{\left(G^{(1)}, \mathbf{I}\right)} \stackrel{\exp }{\rightarrow} G^{(1)}$, $\xi \rightarrow \exp (\xi)=\sum_{j=0}^{\infty} \frac{\xi^{j}}{j !}$, see [B.K.1, §3.5]. This map sends an infinitesimal family of tangent vectors, $\xi(t)=\sum \xi_{j} t^{j} \in T_{\left(G^{(1)}, \mathbf{I}\right)}[[t]]$, to infinitesimal family of group elements, $\exp (\xi(t))$. Denote the collection of the later families by $G[[t]]$.

Definition 3.21. $\quad$ i. An element $\hat{v} \in \hat{F}$ is called $R$-algebraizable if $\hat{v} \in$ Gv for some $v \in F$.

ii. An element $v \in \hat{F}$ is called stably-R-algebraizable if there exists $N \in \mathbb{N}$ such that for any deformation $\hat{v}+\hat{u}(t)$, with $\hat{u}(t) \in$ $(t) \cdot \mathfrak{m}^{N} \cdot \hat{F}[[t]]$, there exists a family $g(t) \in G[[t]]$ satisfying $g(t)(\hat{v}+\hat{u}(t)) \in F[[t]]$, and, moreover, if $\hat{v} \in F$ then one can assume $g(0)=\mathbb{I}$. 
These algebraizability notions depend on the choice of $R$, the strongest notion is for $R=\mathbb{k}[\underline{x}]_{I}$.

Finite determinacy is stronger than $\mathbb{k}[x]]_{I}$-algebraizability and is equivalent to the stable $\mathbb{k}[\underline{x}]_{I}$-algebraizability.

Proposition 3.22. Let $G \circlearrowright \hat{F}$ be an action of a Lie-type group, suppose $G$ is complete with respect to the filtration $\left\{G^{(i)}\right\}$ and for any $\hat{v} \in \hat{F}$ the tangent space $T_{(G \hat{v}, \hat{v})}$ is an $\widehat{R}$-module. Then $\hat{v} \in \hat{F}$ is G-finitely determined iff $\hat{v}$ is stably-R-algebraizable.

Proof. $\Rightarrow \mathrm{As} \hat{v}$ is finitely determined, we can cut off its Tailor tail, thus we assume $\hat{v} \in F$, and denote it by $v$. Choose any $N$ bigger than the $G$-order of determinacy of $v$ and take any deformation $\hat{u}(t)=\sum_{i \geq 1} \hat{u}_{i} t^{i}$, with $\hat{u}_{i} \in \mathfrak{m}^{N} \cdot \hat{F}$. Thus $\left.\hat{u}_{i} \in T_{\left(G^{(1)}\right.} v, v\right)$ and we fix some $\widetilde{\xi}_{i} \in T_{\left(G^{(1)} v, v\right)}$ satisfying $\widetilde{\xi}_{i}(v)=u_{i}$. Thus

$$
v+\sum_{i \geq 1} u_{i} t^{i}=\left(\mathbb{I}+\sum_{i \geq 1} \widetilde{\xi}_{i} t^{i}\right) v=\exp \left(\ln \left(\mathbb{I}+\sum_{i \geq 1} \widetilde{\xi}_{i} t^{i}\right)\right) v=\exp \left(\sum_{i \geq 1} \xi_{i} t^{i}\right) v .
$$

Here $\left\{\xi_{i}\right\}_{i}$ are the elements of $T_{\left(G^{(1)}, \mathbf{I}\right)}$ determined by $\left\{\widetilde{\xi}_{i}\right\}_{i}$. Then $\exp \left(\sum_{i \geq 1} \xi_{i} t^{i}\right) \in G[[t]]$ is the needed element.

$\Leftarrow$ We prove $T_{\left(G^{(1)} v, v\right)} \supseteq \mathfrak{m}^{N} \cdot \hat{F}$, by Theorem 3.18 this implies the finite determinacy.

We can assume $\hat{v} \in F$, thus denote it by $v$. By the assumption, the family $(v+\hat{u} \cdot t) \in \hat{F}[[t]]$, with $\hat{u} \in \mathfrak{m}^{N} \cdot \hat{F}$, is presentable

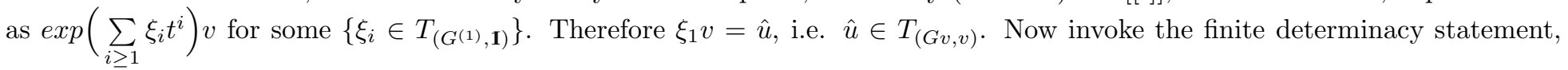
Theorem 3.18 .

Note that in this proof we could not just substitute $t=1$ as the element $\exp \left(\sum \xi_{i}\right)$ is not well defined.

\section{Proofs, examples AND COROLlaries}

4.1. The case of $G_{l}, G_{r}, G_{l r} \circlearrowright \operatorname{Mat}_{m \times n}(R)$.

4.1.1. Approximation of ann $\left(T_{(\Sigma, G, A)}^{1}\right)$.

Proof of Theorem 2.2

1. Note that $T_{\left(G_{r} A, A\right)}=\operatorname{Span}_{R}(A U)_{U \in M a t_{n \times n}(R)}=\bigoplus_{i=1}^{n} \operatorname{Im}(A)=\operatorname{Im}(A)^{\oplus n}$, where $\operatorname{Im}(A) \subset R^{\oplus m}$. In addition, $T_{(\Sigma, A)}=$ $\bigoplus_{i=1}^{n} R^{\oplus m}$. Finally, the two decompositions are compatible, i.e. $T_{(\Sigma, A)} /_{\left(G_{r} A, A\right)}=\bigoplus_{i=1}^{n} R^{\oplus m} / \operatorname{Im}(A)=\bigoplus_{i=1}^{n} \operatorname{coker}(A)$.

2. The $\left(G_{l}, A\right)$ case is identical to the $\left(G_{r}, A^{t}\right)$ case. If $m<n$ then ann.coker $\left(A^{t}\right)=\{0\}$, by Lemma 3.4.

Similarly for $m=n$ : $\operatorname{ann}\left(T_{\left(\Sigma, G_{l}, A\right)}^{1}\right)=\operatorname{ann}\left(T_{\left(\Sigma, G_{r}, A^{t}\right)}^{1}\right)=\operatorname{ann} . \operatorname{coker}\left(A^{t}\right)=\operatorname{ann.coker}(A)$, again, Lemma 3.4.

3. As $G_{l r} \supset G_{r}$, we have $T_{\left(G_{l r} A, A\right)} \supseteq T_{\left(G_{r} A, A\right)}$, thus part 1 gives the bound

$$
\operatorname{ann}\left(T_{\left(\Sigma, G_{l r}, A\right)}^{1}\right) \supseteq \operatorname{ann}\left(T_{\left(\Sigma, G_{r}, A\right)}^{1}\right)=\operatorname{ann.coker}(A) \text {. }
$$

Suppose $f \in \operatorname{ann}\left(T_{\left(\Sigma, G_{l r}, A\right)}^{1}\right)$, i.e.

$$
f \cdot \operatorname{Mat}_{m \times n}(R) \subseteq \operatorname{Span}_{R}\left(U A+A V \mid(U, V) \in \operatorname{Mat}_{m \times m}(R) \times \operatorname{Mat}_{n \times n}(R)\right) .
$$

For any projection onto a discrete valuation domain, $R \stackrel{\phi}{\rightarrow} S$, we get:

$$
\phi(f) \cdot \operatorname{Mat}_{m \times n}(S) \subseteq \operatorname{Span}_{S}\left(U \phi(A)+\phi(A) V \mid(U, V) \in \operatorname{Mat}_{m \times m}(S) \times \operatorname{Mat}_{n \times n}(S)\right) .
$$

Now we use the canonical form, Proposition 3.2, $\phi(A)=P D_{\phi(A)} Q$, where $D_{\phi(A)}$ has zeros off the main diagonal. We get:

$$
P^{-1} \phi(f) \cdot \operatorname{Mat}_{m \times n}(S) Q^{-1} \subseteq \operatorname{Span}_{S}\left(U D_{\phi(A)}+D_{\phi(A)} V \mid(U, V) \in \operatorname{Mat}_{m \times m}(S) \times \operatorname{Mat}_{n \times n}(S)\right) .
$$

On the right hand side one has the space of matrices whose $(m, m)$ 'th component lies in the ideal $\left(\lambda_{m}\right)$. On the left hand side one has $\operatorname{Mat}_{m \times n}(\phi(f))$. Therefore, for $I_{m-1}(\phi(A)) \neq\{0\}$ :

$$
\phi(f) \in\left(\lambda_{m}\right)=\left(I_{m}(\phi(A)): I_{m-1}(\phi(A))\right)=\left(\phi\left(I_{m}(A)\right): \phi\left(I_{m-1}(A)\right)\right) .
$$

Equivalently one has: $\phi\left(f \cdot I_{m-1}(A)\right) \subseteq \phi\left(I_{m}(A)\right)$. Note that this embedding holds also when $I_{m-1}(\phi(A))=\{0\}$. As this holds for any $\phi$, we get: $\overline{f \cdot I_{m-1}(A)} \subseteq \overline{I_{m}(A)}$. As this holds for any $f \in \operatorname{ann}\left(T_{\left(\Sigma, G_{l r}, A\right)}^{1}\right)$, we have:

$$
\overline{I_{m-1}(A) \cdot \operatorname{ann}\left(T_{\left(\Sigma, G_{l r}, A\right)}^{1}\right)} \subseteq \overline{I_{m}(A)} \text {. }
$$

Finally, by Lemma 3.8, for $I_{m-1}(A) \neq\{0\}$, we have: $\overline{\operatorname{ann}\left(T_{\left(\Sigma, G_{l r}, A\right)}^{1}\right)} \subseteq \overline{I_{m}(A)}: \overline{I_{m-1}(A)}$.

For $I_{m-1}(A)=\{0\}$ similar arguments show: $\phi\left(\operatorname{ann}\left(T_{\left(\Sigma, G_{l r}, A\right)}^{1}\right)\right)=\{0\}$ for any $R \stackrel{\phi}{\rightarrow} S_{D V R}$.

Remark 4.1. As one sees from the end of this proof, we have a stronger property: $\overline{\operatorname{ann}\left(T_{\left(\Sigma, G_{l r}, A\right)}^{1}\right) \cdot I_{m-1}(A)} \subseteq \overline{I_{m}(A)}$.

One would like to strengthen it further, to achieve from $\phi(f) \in \phi\left(I_{m}(A)\right): \phi\left(I_{m-1}(A)\right)$ that $\phi(f) \in \phi\left(I_{m}(A): I_{m-1}(A)\right)$. Then

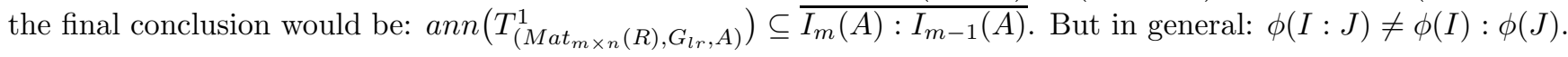

Example 4.2. Let $R$ be a Noetherian ring and $A \in \operatorname{Mat}_{m \times n}(R)$. Suppose that the ideal $I_{m}(A)$ is radical and $h e i g h t\left(I_{m-1}(A)\right)>$ height $\left(I_{m}(A)\right)$. Then ann.coker $(A)=(\operatorname{det}(A))$, see Property $(3.3)$. In addition $I_{m}(A)=\overline{I_{m}(A)}$. Finally, as height $\left(I_{m-1}(A)\right)>$ height $\left(I_{m}(A)\right)$, we have $\overline{I_{m}(A)}=\overline{I_{m}(A)}: \overline{I_{m-1}(A)}$. Altogether we get: $\operatorname{ann}\left(T_{\left(\Sigma, G_{l r}, A\right)}^{1}\right)=(\operatorname{det}(A))$. 
Example 4.3. Suppose $R$ is a PID. Then all ideals are principal, hence integrally closed. Thus, for any $A \in M a t_{m \times n}(R)$ we have

$$
\text { ann.coker }(A)=I_{m}(A): I_{m-1}(A)=\overline{I_{m}(A)}: \overline{I_{m-1}(A)} \text {. }
$$

(For example, bring $A$ to the Smith normal form.) Therefore $\operatorname{ann}\left(T_{\left(\Sigma, G_{l r}, A\right)}^{1}\right)=I_{m}(A): I_{m-1}(A)=\operatorname{ann.coker}(A)$.

\subsubsection{Applications to finite determinacy.}

Proof of Proposition 2.3.

We use Proposition 3.19. First we remark that the assumption (3) ensures the needed approximation property of $R$, see $\S 3.10$. Indeed, in all the cases the equations $U A=A+B, A V^{-1}=A+B, U A V^{-1}=A+B$ are linear in the entries of $U, V$. (In the later case present the equation in the form $U A=(A+B) V$.)

1. Note that $\operatorname{ann}\left(T_{\left(\Sigma, G_{r}^{(m)}, A\right)}^{1}\right)=\operatorname{ann} R^{\oplus m} / \mathfrak{m} \cdot \operatorname{Im}(A)=\operatorname{ann} R^{\oplus m} / \operatorname{Im}\left(\left.A\right|_{\mathfrak{m} \cdot R^{\oplus n}}\right)$. Thus Proposition 3.19 gives the stated bound on $\operatorname{ord}_{G_{r}}^{\Sigma}(A)$.

i. If $\operatorname{dim}(R)>(n-m+1)$ then for any $A \in \operatorname{Mat}_{m \times n}(\mathfrak{m})$ the ideal ann.coker $(A)$, having height at most $(n-m+1)$, see Property (3.3), cannot contain any power of the maximal ideal $\mathfrak{m}$. This implies the statement.

ii. Vice versa, the height of the ideal ann.coker $(A)$ is generically the expected one, see $\S 3.2$. Thus for any $A$ and the generic $B \in \operatorname{Mat}_{m \times n}\left(\mathfrak{m}^{N}\right)$, the ideal ann.coker $(A+B)$ is of height $\min ((n-m+1), \operatorname{dim}(R))$. Thus if $\operatorname{dim}(R) \leq(n-m+1)$ then ann.coker $(A+B)$ contains a (finite) power of the maximal ideal.

2. Follows immediately from part 2 of Theorem 2.2 .

3. i. Note that height $\left(\overline{I_{m}(A)}: \overline{I_{m-1}(A)}\right)=$ height $\left(\overline{I_{m}(A)}\right)=$ height $\left(I_{m}(A)\right)=$ height $($ ann.coker $(A))$. Thus the statement follows immediately from the first part and part 3 of Theorem 2.2.

3.ii. Note that $G_{l r} \supset G_{r}$, thus, by part 1:

$$
\operatorname{ord}_{G_{l r}}^{\Sigma}(A) \leq \operatorname{ord}_{G_{r}}^{\Sigma}(A) \leq l l_{R}\left(\text { ann.coker }\left(\left.A\right|_{\mathfrak{m} R^{p}}\right)\right)-1 .
$$

If $R$ is Noetherian then the lower bound on $\operatorname{ord}_{G_{l r}}^{\Sigma}(A)$ is immediate.

To prove the lower bound in the non-Noetherian case we use the surjectivity of the completion, $R \stackrel{\hat{\psi}}{\rightarrow} \widehat{R}$. Then by Lemma 3.15 we have the equality of Loewy lengths:

$$
l l_{R}\left(\operatorname{ann}\left(T_{\left(\Sigma, G_{l r}, A\right)}^{1}\right)\right)=l l_{\widehat{R}}\left(\hat{\psi}\left(\operatorname{ann}\left(T_{\left(\Sigma, G_{l r}, A\right)}^{1}\right)\right)\right)=l l_{\widehat{R}}\left(\operatorname{ann} \hat{\psi}\left(T_{(\Sigma, A)}\right) \hat{\psi}\left(T_{\left(G_{l r} A, A\right)}\right)\right)=l l_{\widehat{R}}\left(\overline{I_{m}(\widehat{A})}: \overline{I_{m-1}(\widehat{A})}\right)
$$

Example 4.4. Consider the trivial case: $A$ is a "numerical" matrix (with entries in $\mathbb{k}$ ), $m \leq n$. Then ann.coker $(A)$ is either $R$ or $\{0\}$. Hence $A$ is finitely $G_{l r}$-determined iff at least one of its maximal minors is a non-zero constant, i.e. $A$ is of the full rank. (In other words, $A$ is invertible from the left, i.e. it has no left-kernel.) In this case, for $m \leq n, A$ is 0 -determined with respect to $G_{r}$.

Example 4.5. Let $R=\mathbb{k}[[x, y]]$ and $A=\left(\begin{array}{ccc}x^{5} & 0 & y^{3} \\ 0 & y^{4} & x^{3}\end{array}\right)$. Then $I_{1}(A)=\left(x^{3}, y^{3}\right), I_{2}(A)=\left(y^{7}, x^{5} y^{4}, x^{8}\right)$ and ann.coker $(A)=$ $\left(y^{7}, x^{5} y^{4}, x^{8}\right)$. (To compute ann.coker one can notice that height $\left(I_{2}(A)\right)=2$, as expected, thus ann.coker $(A)=I_{2}(A)$, by Property (3.3).) Therefore $\overline{I_{1}(A)}=\mathfrak{m}^{3}$ and $\overline{I_{2}(A)}=\mathfrak{m}^{8}+\left(y^{7}\right)$. Thus

$$
\left(y^{7}, x^{5} y^{4}, x^{8}\right) \subseteq \operatorname{annT}_{\left(\Sigma, G_{l r}, A\right)}^{1} \subseteq \overline{I_{2}(A)}: \overline{I_{1}(A)}=\mathfrak{m}^{5} .
$$

Note that ann.coker $\left(\left.A\right|_{\mathfrak{m} \cdot R^{\oplus 3}}\right)=\left(y^{7}, x^{5} y^{4}, x^{8}\right)$. Finally, $l l_{R}\left(\mathfrak{m}^{5}\right)=5$ and $l l_{R}\left(y^{7}, x^{5} y^{4}, x^{8}\right)=11$. We get: $4 \leq$ ord $d_{G_{l r}}^{\Sigma}(A) \leq 10$.

Example 4.6. Suppose $R$ satisfies the assumption $(3)$ and $\operatorname{dim}(R)>0$. Let $\Sigma=\operatorname{Mat}_{m \times n}(R)$.

1. For $m=n, A \in M a t_{m \times m}(\mathfrak{m})$ is finitely- $G_{l r}$-determined iff $\operatorname{dim}(R)=1, \operatorname{det}(A) \in R$ is not a zero $\operatorname{divisor}$ and $\operatorname{det}(A) \notin \mathfrak{m}^{\infty}$. This generalizes Part 1 of [Bruce-Tari, Theorem 1.1].

Proof: for square matrices height $(\operatorname{ann} . \operatorname{coker}(A))=\operatorname{height}(\operatorname{det}(A)) \leq 1$. Thus it can contain a power of the maximal ideal only when $\operatorname{dim}(R) \leq 1$. Now invoke Proposition 2.3 .

2. Suppose $\operatorname{dim}(R)=2$ and $m<n$. Suppose $A$ has at least two $m \times m$ blocks whose determinants, $\Delta_{1}, \Delta_{2}$, are neither zero divisors, nor belong to $\mathfrak{m}^{\infty}$ and moreover are relatively prime, i.e. if $\Delta_{i}=a_{i} h \in R$ then $h \in R$ is invertible. Then A is finitely $G_{r}$-determined.

Proof: Let $\Delta_{1}, \Delta_{2}$ be two such determinants then the height of the ideal $\left(\Delta_{1}\right)+\left(\Delta_{2}\right)$ is two. Thus height $($ ann.coker $(A))=2$ and ann.coker $(A)$ contains a power of $\mathfrak{m}$.

Example 4.7. Suppose $R$ satisfies the assumption (3) and $A \in \operatorname{Mat}_{m \times n}(R)$. Then part 3 of Theorem 2.2 and Theorem 3.18 imply:

$$
\text { if } \widetilde{A} \equiv A \bmod (\mathfrak{m} \cdot \operatorname{ann} \cdot \operatorname{coker}(A)) \text { then } \widetilde{A} \stackrel{G_{l r}}{\sim} A \text {. }
$$

This both strengthens (quantifies) and generalizes [Cutkosky-Srinivasan, Theorem 5.2].

4.1.3. Applications to the infinite determinacy. Part 1 of Proposition 2.3 implies the following criterion.

Corollary 4.8. Let $A \in \operatorname{Mat}_{m \times n}\left(C^{\infty}\left(\mathbb{R}^{p}, 0\right)\right)$. If there exists an element $f \in I_{m}(A)$ satisfying Eojasiewicz inequality, Equation (26), then $A$ is infinitely-G-determined.

Indeed, by Proposition 2.3 it is enough to check that the ideal $I_{m}(A)$ contains $\mathfrak{m}^{\infty}$, in other words: any function flat at the origin is divisible by some element of $I_{m}(A)$. And this is ensured by Łojasiewicz inequality. 
4.2. (Anti-)symmetric matrices and the congruence.

\subsubsection{Computation of ann $\left(T_{\left(\Sigma, G_{c o n g r}, A\right)}^{1}\right)$. Proof of Theorem 2.4.}

1. First we assume that $R$ is Noetherian. To prove that $\operatorname{ann}\left(T_{\left(\Sigma, G_{\text {congr }}, A\right)}^{1}\right)=\{0\}$ it is enough to show that this module is not a torsion module and is supported at the generic point of any component of $\operatorname{Spec}(R)$. Thus we assume that $\operatorname{Spec}(R)$ is irreducible and localize at the generic point of $\operatorname{Spec}(R)$. (Note that $\operatorname{dim}(R)>0$.) At this point we compare the modules, or rather vector spaces, $\left(T_{(G A, A)}\right)_{(0)}$ and $\left(T_{(\Sigma, A)}\right)_{(0)}$.

Note that $R_{(0)}$ is a field, thus we can bring the symmetric part of $A$ to the canonical form of Proposition 3.2 , i.e. $A \stackrel{G_{\text {congr }}}{\sim}$ Diag $+A_{-}$, where Diag is diagonal while $A_{-} \in M a t_{m \times m}^{\text {skew-sym }}(R)$. We can assume that $A+A^{t}$ is generically non-degenerate on $\operatorname{Spec}(R)$, therefore Diag is invertible over $R_{(0)}$. To understand $\left(T_{(G A, A)}\right)_{(0)}$, which is spanned by $U A+A U^{t}$, we re-scale the matrices, $\tilde{U}:=U \cdot \operatorname{Diag}$, and expand them into the symmetric and skew-symmetric parts, $\tilde{U}=\tilde{U}_{+}+\tilde{U}_{-}$. Then $\left(T_{(G A, A)}\right)_{(0)}=$ $\operatorname{Span}_{R}\left(2 \tilde{U}_{+}, \tilde{U} A_{-}+A_{-} \tilde{U}^{t}\right)$. To prove that

$$
\left(T_{(G A, A)}\right)_{(0)} \subsetneq\left(T_{(\Sigma, A)}\right)_{(0)}=M a t_{m \times m}^{\text {sym }}\left(R_{(0)}\right) \oplus M a t_{m \times m}^{\text {skew-sym }}\left(R_{(0)}\right)
$$

we consider the map

$$
\operatorname{Mat}_{m \times m}\left(R_{(0)}\right) \stackrel{\phi}{\rightarrow} M a t_{m \times m}^{s y m}\left(R_{(0)}\right) \oplus M a t_{m \times m}^{\text {skew-sym }}\left(R_{(0)}\right), \quad \tilde{U} \rightarrow\left(2 \tilde{U}_{+}, \tilde{U} A_{-}+A_{-} \tilde{U}^{t}\right)
$$

Note that the domain and the target are $R_{(0)}$-vector spaces of the same (finite) dimension. Thus it is enough to demonstrate a non-trivial kernel of $\phi$. Indeed, the space $\operatorname{ker}(\phi)=\left\{\tilde{U}_{+}=\mathbb{O}, \tilde{U}_{-} A_{-}=A_{-} \tilde{U}_{-}\right\}$is non-zero. It contains all the matrices $\tilde{U}_{-}$that commute with $A_{-}$, e.g. $\operatorname{ker}(\phi) \supseteq \operatorname{Span}_{R}\left(A_{-}, A_{-}^{3}, A_{-}^{5}, \ldots\right)$.

Now, if $R$ is non-Noetherian, but satisfies condition (3), we use Lemma 3.15 to get: $\hat{\psi}\left(\operatorname{ann}\left(T^{1}\right)\right)=\{0\}$. Therefore $\operatorname{ann}\left(T^{1}\right) \subseteq$ $\mathfrak{m}^{\infty}$.

2. To prove that $\operatorname{ann}\left(T_{\left(\Sigma, G_{\text {congr }}, A\right)}^{1}\right) \supseteq \operatorname{ann.coker}(A)$, take any $B \in M a t_{m \times m}^{\text {sym }}$ (ann.coker $\left.(A)\right)$. By the definition of the annihilator-of-cokernel there exists $U$ satisfying: $A U=\frac{B}{2}$. As $A, B$ are symmetric: $U^{t} A=\frac{B}{2}$. Therefore $U A+A U^{t}=B$. But $U A+A U^{t} \in T_{\left(G_{\text {congr }} A, A\right)}$, see Example 3.13. Thus ann.coker $(A) \cdot T_{(\Sigma, A)}=M a t_{m \times m}^{\text {sym }}(\operatorname{ann} . \operatorname{coker}(A)) \subseteq T_{\left(G_{\text {congr }} A, A\right)}$.

To prove that $\operatorname{ann}\left(T_{\left(\Sigma, G_{\text {congr }}, A\right)}^{1}\right) \subseteq \overline{I_{m}(A)}: \overline{I_{m-1}(A)}$, let $f \in \operatorname{ann}\left(T_{\left(\Sigma, G_{\text {congr }}, A\right)}^{1}\right)$, i.e. $f \cdot M a t_{m \times m}^{s y m}(R) \subseteq S p a n_{R}\left(U A+A U^{t} \mid U \in\right.$ $\operatorname{Mat}_{m \times m}(R)$. Then, for any $R \stackrel{\phi}{\rightarrow} S_{D V R}$ :

$$
\phi(f) \cdot \operatorname{Mat}_{m \times m}^{\text {sym }}(S) \subseteq \operatorname{Span}_{R}\left(U \phi(A)+\phi(A) U^{t} \mid U \in M a t_{m \times m}(S)\right) .
$$

Now, bring $\phi(A)$ to the canonical form (using Proposition 3.2), $\phi(A)=V \cdot D_{\phi(A)} V^{t}$, where $D_{\phi(A)}=\oplus \lambda_{i} \mathbb{I}$, with $\left(\lambda_{1}\right) \supseteq\left(\lambda_{2}\right) \supseteq$ $\cdots \supseteq\left(\lambda_{m}\right)$. Therefore:

$$
\phi(f) \cdot \operatorname{Mat}_{m \times m}^{\text {sym }}(S) \subseteq \operatorname{Span}_{R}\left(U D_{\phi(A)}+D_{\phi(A)} U^{t} \mid U \in M a t_{m \times m}(S)\right) .
$$

But the $(m, m)$-entry of any matrix of the form $\left(U D_{\phi(A)}+D_{\phi(A)} U^{t}\right)$ lies in the ideal

$$
\left(\lambda_{m}\right)=\operatorname{ann.coker}(\phi(A))=I_{m}(\phi(A)): I_{m-1}(\phi(A))=\phi\left(I_{m}(A)\right): \phi\left(I_{m-1}(A)\right) .
$$

Therefore $\phi\left(f \cdot I_{m-1}(A)\right) \in \phi\left(I_{m}(A)\right)$. As this holds for any $R \stackrel{\phi}{\rightarrow} S_{D V R}$ we get: $\overline{f \cdot I_{m-1}(A)} \subseteq \overline{I_{m}(A)}$. Finally, Equation $(20)$ implies $f \in \overline{I_{m}(A)}: \overline{I_{m-1}(A)}$.

3. First we prove the part $(\cdots) \subseteq \operatorname{ann}\left(T_{\left(\Sigma, G_{\text {congr }}, A\right)}^{1}\right)$.

3.i. For $m$-even the proof of $\operatorname{ann}\left(T_{\left(M a t_{m \times m}^{\text {skew-sym }}(R), G_{\text {congr }}, A\right)}^{1}\right) \supseteq \operatorname{ann.coker}(A)$ goes as in part 2.

3.ii. Suppose $m$ is odd, then by Proposition 3.5: $T_{\left(G_{\text {congr }} A, A\right)} \supseteq M a t_{m \times m}^{\text {skew-sym }}\left(P f_{m-1}(A)\right)$. Thus ann $\left(T_{\left(\Sigma, G_{\text {congr }}, A\right)}^{1}\right) \supseteq$ $\left(P f_{m-1}(A)\right)$.

To establish the bound $\operatorname{ann}\left(T_{\left(\Sigma, G_{\text {congr }}, A\right)}^{1}\right) \subseteq(\cdots)$, let $f \in \operatorname{ann}\left(T_{\Sigma, G_{\text {congr }}, A}^{1}\right)$, i.e. $f \cdot M a t_{m \times m}^{\text {skew-sym }}(R) \subseteq S p a n_{R}\left(U A+A U^{t} \mid U \in\right.$ $\operatorname{Mat}_{m \times m}(R)$. Then, for any $R \stackrel{\phi}{\rightarrow} S_{D V R}$ :

$$
\phi(f) \cdot \operatorname{Mat}_{m \times m}^{\text {skew-sym }}(S) \subseteq \operatorname{Span}_{R}\left(U \phi(A)+\phi(A) U^{t} \mid U \in \operatorname{Mat}_{m \times m}(S)\right) .
$$

Bring $\phi(A)$ to the canonical form (using Proposition 3.2), $\phi(A)=V \cdot E_{\phi(A)} V^{t}$, where $E_{\phi(A)}=\oplus_{i} \lambda_{i} E_{i}$ and $\left(\lambda_{1}\right) \supseteq\left(\lambda_{2}\right) \cdots \supseteq\left(\lambda_{\left\lfloor\frac{m}{2}\right\rfloor}\right)$. Therefore:

$$
\phi(f) \cdot \operatorname{Mat}_{m \times m}^{\text {skew }- \text { sym }}(S) \subseteq \operatorname{Span}_{R}\left(U E_{\phi(A)}+E_{\phi(A)} U^{t} \mid U \in \operatorname{Mat}_{m \times m}(S)\right) .
$$

The $(m-1, m)$ and $(m, m-1)$ entries of $\left(U E_{\phi(A)}+E_{\phi(A)} U^{t}\right)$ belong to the ideal $\left(\lambda_{\left\lfloor\frac{m}{2}\right\rfloor}\right)$. Therefore,

$$
\phi(f) \in\left(\lambda_{\left\lfloor\frac{m}{2}\right\rfloor}\right)=I_{m-1}(\phi(A)): I_{m-2}(\phi(A))=\phi\left(I_{m-1}(A)\right): \phi\left(I_{m-2}(A)\right),
$$

i.e. $\phi\left(f \cdot I_{m-2}(A)\right) \subseteq \phi\left(I_{m-1}(A)\right)$.

As this holds for any $R \stackrel{\phi}{\rightarrow} S_{D V R}$ we get: $\overline{f \cdot I_{m-2}(A)} \subseteq \overline{I_{m-1}(A)}$. Finally, we get $f \in \overline{I_{m-1}(A)}: \overline{I_{m-2}(A)}$, see Equation $(20)$. Therefore $\operatorname{ann}\left(T_{\left(\Sigma, G_{\text {congr }}, A\right)}^{1}\right) \subseteq \overline{I_{m-1}(A)}: \overline{I_{m-2}(A)}$.

Finally, if $m$ is even, then $\phi\left(I_{m}(A)\right): \phi\left(I_{m-1}(A)\right)=\phi\left(I_{m-1}(A)\right): \phi\left(I_{m-2}(A)\right)$. Thus in Equation $(51)$ we have: $\phi(f) \in$ $\phi\left(I_{m}(A)\right): \phi\left(I_{m-1}(A)\right)$ and therefore $\operatorname{ann}\left(T_{\left(M a t_{m \times m}^{\text {skew-sym }}(R), G_{\text {congr }}, A\right)}^{1}\right) \subseteq \overline{I_{m}(A)}: \overline{I_{m-1}(A)}$. 
Example 4.9. (Continuing examples 4.2 and 4.3.) In some cases the bounds on $\operatorname{ann}\left(T_{\left(\Sigma, G_{l r}, A\right)}^{1}\right)$ turn into equality. Let $R$ be Noetherian ring and $A$ either symmetric or skew-symmetric of even size. Suppose

i. either $R$ is PID

ii. or $I_{m}(A)$ is radical and height $\left(I_{m-1}(A)\right)>$ height $\left(I_{m}(A)\right)>0$.

Then $\operatorname{ann}\left(T_{\left(\Sigma, G_{l r}, A\right)}^{1}\right)=$ ann.coker $(A)$. Indeed, $\overline{I_{m}(A)}=I_{m}(A)$ (Property 3.6) and ann.coker $(A)=I_{m}(A): I_{m-1}(A)(\operatorname{Property}$ $3.4)$.

Example 4.10. In the low dimensional cases $T_{\left(\Sigma, G_{c o n g r}, A\right)}^{1}$ is easily written down explicitly.

1. In the trivial case $A=\left(\begin{array}{cc}0 & a \\ -a & 0\end{array}\right)$ we get ann.coker $(A)=(a) \subset R$ and thus $T_{\left(\Sigma, G_{\text {congr }}, A\right)}^{1} \approx(R /(a))^{\oplus 2}$.

2. For $3 \times 3$ matrices take $A=\left(\begin{array}{ccc}0 & a & b \\ -a & 0 & c \\ -b & -c & 0\end{array}\right)$, matrix of indeterminates, we get

$$
\left(P f_{2}(A)\right)=(a)+(b)+(c)=\overline{I_{2}(A)}: \overline{I_{1}(A)} \subset R .
$$

Therefore $T_{\left(\Sigma, G_{\text {congr }}, A\right)}^{1} \approx(R /(a)+(b)+(c))^{\oplus 3}$.

4.2.2. Finite determinacy for congruence.

Proof of Proposition 2.5. The statements 1, 1', 1" follow from Theorem 2.4 because height $($ ann.coker $(A)) \leq 1$ for a square matrix, while for a skew-symmetric matrix of odd-size height $\left(P f_{m-1}(A)\right) \leq 3$, see $\S 3.2$.

The statements 2.i. 2.ii. follow from Theorem 2.4 and Proposition 3.19.

Example 4.11. The natural question is the order of determinacy for a generic (skew-)symmetric matrix. By Proposition 2.5 we must assume $\operatorname{dim}(R)=1$. For simplicity we assume that $R$ is a Henselian DVR.

i. Take the generic matrix $A \in M a t_{m \times m}^{s y m}\left(\mathfrak{m}^{k}\right)$. Use Proposition 3.2 to bring $A$ to the canonical form. (Note that the order of determinacy and $\operatorname{ann}\left(T_{\left(\Sigma, G_{\text {congr }}, A\right)}^{1}\right)$ are invariant under the $G_{\text {congr-action.) }}$ Thus $A=\underset{j}{\oplus} \lambda_{j} \mathbb{I}$ and by genericity the orders of all $\lambda_{j}$ are $k$. Thus the order of determinacy is $k$. We get the rigidity in the following sense. Denote $\Sigma^{(k+1)}=\{A\}+$ $M a t_{m \times m}^{s y m}\left(\mathfrak{m}^{k+1}\right)$, then $T_{\left(\Sigma^{(k+1)}, G_{\text {congr }}, A\right)}^{1}=\{0\}$.

ii. Similarly for skew-symmetric matrices of even size. Let $A \in M a t_{m \times m}^{\text {skew-sym }}\left(\mathfrak{m}^{k}\right)$ be generic, then $A \stackrel{G_{c o n g r}}{\sim} \underset{j}{\oplus} \lambda_{j} E_{j}$, where $\operatorname{ord}\left(\lambda_{j}\right)=k$. Thus the order of determinacy is $k$. If one takes $\Sigma^{(k+1)}=\{A\}+M a t_{m \times m}^{\text {skew-sym }}\left(\mathfrak{m}^{k+1}\right)$ then $\left.T_{(\Sigma(k+1)}^{1}, G_{c o n g r}, A\right)=$ $\{0\}$.

Proposition 2.5 implies the following criterion for the infinite determinacy of (skew-)symmetric forms valued in the germs of smooth functions.

Corollary 4.12. 1. Let either $A \in M a t_{m \times m}^{\text {sym }}\left(C^{\infty}\left(\mathbb{R}^{p}, 0\right)\right)$ or $A \in M a t_{m \times m}^{\text {skew-sym }}\left(C^{\infty}\left(\mathbb{R}^{p}, 0\right)\right)$, for $m$-even. If det $(A)$ satisfies the Lojasiewicz inequality, Equation (26), then $A$ is infinitely-G $G_{\text {congr-determined. }}$

2. Let $A \in M a t_{m \times m}^{\text {skew-sym }}\left(C^{\infty}\left(\mathbb{R}^{p}, 0\right)\right)$, for $m$-odd. If there exists an element $f \in I_{m-1}(A)$ that satisfies the Eojasiewicz inequality, then $A$ is infinitely- $G_{\text {congr-determined. }}$

The argument is the same as for Corollary 4.8 .

4.3. The upper-block-triangular matrices and the action of $G_{r}^{u p}, G_{l r}^{u p}$. First we give two examples showing that the block-triangular matrices appear naturally, then we prove Theorem 2.6.

4.3.1. Morphisms of filtered free modules and their deformations. Let $M$ be a free module and denote by $M_{\bullet}$ a filtration by free

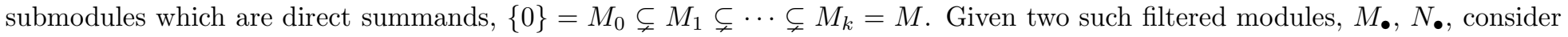
their filtered homomorphisms, $\operatorname{Hom}_{R}\left(M_{\bullet}, N_{\bullet}\right)=\left\{\phi \in \operatorname{Hom}_{R}(M, N) \mid \phi\left(M_{i}\right) \subseteq N_{i}\right\}$. Fix a filtered basis of $M$, i.e. a minimal set of generators of $M$ compatible with the filtration:

$$
\left(e_{1}, \ldots, e_{i_{1}}, e_{i_{1}+1}, \ldots, e_{i_{2}}, \ldots, e_{i_{k-1}+1}, \ldots, e_{i_{k}}\right), \quad\left\{M_{j}=\operatorname{Span}_{R}\left(e_{1}, \ldots, e_{i_{j}}\right)\right\}_{j=1, \ldots, k} .
$$

Similarly fix an $N_{\bullet}$-basis. For these chosen bases any homomorphism $\phi \in \operatorname{Hom}_{R}\left(M_{\bullet}, N_{\bullet}\right)$ is presented by an upper-block-triangular matrix,

$$
[\phi]=A=\left(\begin{array}{ccccc}
A_{11} & A_{12} & \ldots \ldots & \ldots & A_{1 k} \\
\mathbb{O} & A_{22} & \ldots & \ldots & A_{2 k} \\
\ldots & \ldots & \ldots & \ldots & \ldots \\
\mathbb{O} & \mathbb{O} & \ldots & \mathbb{O} & A_{k k}
\end{array}\right) .
$$

The change of bases in $M_{\bullet}, N_{\bullet}$ act on $A$ by elements of $G_{l r}^{u p}=G_{l}^{u p} \times G_{r}^{u p}=G L\left(M_{\bullet}, R\right) \times G L\left(N_{\bullet}, R\right)$. Thus for the deformations of $\phi$ inside $\Sigma=\operatorname{Hom}_{R}\left(M_{\bullet}, N_{\bullet}\right)$ we have:

$$
T_{\left(\Sigma, G_{l r}^{u p}, A\right)}^{1}=T_{\phi \in \operatorname{Hom}_{R}\left(M_{\bullet}, N_{\bullet}\right)}^{1}=T_{\left(M a t^{u p}, A\right)} / T_{\left(G_{l r}^{u p} A, A\right)} .
$$


4.3.2. Deformations of filtered submodules. Fix a filtered module $\{0\}=M_{0} \subsetneq M_{1} \subsetneq \cdots \subsetneq M_{k}=M \subseteq R^{\oplus m}$, not necessarily free. Suppose a sequence of "containers" is prescribed, i.e. $M_{i} \subseteq V_{i}$, where $V_{i} \subseteq R^{\oplus m}$ is a free direct summand. We assume $V_{i} \subsetneq V_{i+1}$. Choose a set of generators of $R^{\oplus m}$ compatibly with the filtration $V_{i} \subsetneq V_{i+1} \subsetneq \ldots R^{\oplus m}$. Then the generating matrix $A_{M}$ of $M$ is upper-block-triangular:

$$
A_{M}=\left(\begin{array}{ccccc}
A_{11} & A_{12} & \ldots \ldots & \ldots & A_{1 k} \\
\mathbb{O} & A_{22} & \ldots & \ldots & A_{2 k} \\
\ldots & \ldots & \ldots & \ldots & \ldots \\
\mathbb{O} & \mathbb{O} & \ldots & \mathbb{O} & A_{k k}
\end{array}\right) .
$$

A change of basis of $M_{\bullet}$ corresponds to the $G_{r}^{u p}$-action. Therefore for the deformations of $M_{\bullet}$, inside the ambient filtration $V_{\bullet}$, we have

$$
T_{\left(\Sigma, G_{r}, A\right)}^{1}=T_{M_{\bullet}, V_{\bullet}}^{1}=T_{\left(M a t^{u p}, A\right)} / T_{\left(G_{r}^{u p} A, A\right)}
$$

4.3.3. Proof of Theorem 2.6.

1. Denote $\Sigma=M a t_{\left\{m_{i}\right\} \times\left\{n_{j}\right\}}^{u p}(R)$, thus $T_{(\Sigma, A)} \approx M a t_{\left\{m_{i}\right\} \times\left\{n_{j}\right\}}^{u p}(R)$. We write down the tangent space to the group orbit:

$$
T_{\left(G_{r}^{u p} A, A\right)}=\operatorname{Span}_{R}\left(\left(\begin{array}{cccc}
A_{11} U_{11} & A_{11} U_{12}+A_{12} U_{22} & \ldots & \ldots \sum_{i=1}^{k} A_{1 i} U_{i k} \\
\mathbb{O} & A_{22} U_{22} & \ldots & \sum_{i=2}^{k} A_{2 i} U_{i k} \\
\ldots & \ldots & \ldots & \ldots \\
\mathbb{O} & \ldots & \mathbb{O} & A_{k k} U_{k k}
\end{array}\right),\left\{U_{i j} \in \operatorname{Mat}_{m_{i} \times n_{j}}(R)\right\}_{i \leq j}\right)
$$

Note that both modules $T_{(\Sigma, A)}$ and $T_{\left(G_{r}^{u p} A, A\right)}$ decompose into the direct sums, according to the blocks of columns. Explicitly, $T_{(\Sigma, A)}=\bigoplus_{l=1}^{k} \Lambda_{l}$, where $\Lambda_{l}$ consists of all matrices $B$ with the block-structure $B=\left\{B_{i j}\right\}$ satisfying: $B_{i j}=\mathbb{O}$ if $j \neq l$ or if $i>j$.

Therefore, for a fixed $A$ we have: $T_{\left(\Sigma, G_{r}^{u p}, A\right)}^{1}=\bigoplus_{q=1}^{k}\left(T_{\left(\Sigma, G_{r}^{u p}, A\right)}^{1}\right)_{q}$, where

$$
\begin{aligned}
& \left(T_{\left(\Sigma, G_{r}^{u p}, A\right)}^{1}\right)_{q}= \\
& \frac{M a t_{\left(\sum_{j=1}^{q} m_{j}\right) \times n_{q}}(R)}{\operatorname{Span}_{R}\left(\left(\begin{array}{cccc}
A_{11} & \ldots & \ldots & A_{1 q} \\
\mathbb{O} & \ldots & \ldots & \\
\mathbb{O} & \ldots & \mathbb{O} & A_{q q}
\end{array}\right)\left(\begin{array}{c}
U_{1 q} \\
\ldots \\
U_{q q}
\end{array}\right), U_{j q} \in \operatorname{Mat}_{m_{j} \times n_{q}}(R)\right)}=\operatorname{coker}\left(\begin{array}{cccc}
A_{11} & \ldots & \ldots & A_{1 q} \\
\mathbb{O} & \ldots & \ldots & \\
\mathbb{O} & \ldots & \mathbb{O} & A_{q q}
\end{array}\right)=\operatorname{ann.coker}\left(\underline{A \|_{q}}\right) .
\end{aligned}
$$

Accordingly: $\operatorname{ann}\left(T_{\left(\Sigma, G_{r}^{u p}, A\right)}^{1}\right)=\bigcap_{q=1}^{k} \operatorname{ann}\left(T_{\left(\Sigma, G_{r}^{u p}, A\right)}^{1}\right)_{q}=\bigcap_{q=1}^{k} \operatorname{ann} \cdot \operatorname{coker}\left(\underline{A}_{q}\right)$. This proves the first statement.

2. The bounds $\prod_{q=1}^{k}$ ann.coker $\left(A_{q q}\right) \subseteq \bigcap_{q=1}^{k}$ ann.coker $\left(\underline{A} \|_{q}\right) \subseteq \bigcap_{q=1}^{k}$ ann.coker $\left(A_{q q}\right)$ are proved by induction as follows. The case $k=1$ is trivial. Suppose the bounds hold for $(k-1)$. To establish them for $k$ it is enough to prove:

i. ann.coker $(A) \subseteq$ ann.coker $\left(A_{k k}\right)$

ii. ann.coker $(A) \supseteq \prod_{q=1}^{k}$ ann.coker $\left(A_{q q}\right)$.

In the proof we decompose $R^{\sum m_{q}}=\underset{q}{\oplus} R^{m_{q}}$. To keep track of the block structure we denote the image of $R^{m_{q}}$ inside this sum by $R^{m_{q}} \oplus\{0\}$.

Proof of $i$. If $f \in$ ann.coker $(A)$ then $f \cdot\left(\{0\} \oplus R^{m_{k}}\right) \subseteq \operatorname{Im}(A)$ and thus $f \cdot R^{m_{k}} \subseteq \operatorname{Im}\left(A_{k k}\right)$, i.e. $f \in$ ann.coker $\left(A_{k k}\right)$.

Proof of ii. It is enough to check: for any collection $\left\{f_{q} \in \operatorname{ann.coker}\left(A_{q q}\right)\right\}_{q=1 \ldots k}$ holds: $\prod_{q=1}^{k} f_{q} \in \operatorname{ann.coker}(A)$.

By the inductive assumption we have: $\left(\prod_{q=1}^{k-1} f_{q}\right) \cdot\left(\bigoplus_{q=1}^{k-1} R^{m_{q}} \oplus\{0\}\right) \subseteq \operatorname{Im}(A)$. It remains to check: $\left(\prod_{q=1}^{k} f_{q}\right) \cdot\left(\{0\} \oplus R^{m_{k}}\right) \subseteq \operatorname{Im}(A)$. Indeed, any $0 \oplus v \in\left(\prod_{q=1}^{k} f_{q}\right) \cdot\left(\{0\} \oplus R^{m_{k}}\right)$ is presentable as $v=A_{k k} u$ for some $u \in\left(\prod_{q=1}^{k-1} f_{q}\right) R^{n_{k}}$. Thus $v-A(0 \oplus u) \in$ $\left(\prod_{q=1}^{k-1} f_{q}\right) \cdot\left({\underset{\oplus}{q}}^{k-1} R^{m_{q}} \oplus\{0\}\right) \subseteq \operatorname{Im}(A)$

3. The lower bound on $\operatorname{ann}\left(T_{\left(\Sigma, G_{l r}^{u p}, A\right)}^{1}\right)$ follows by parts 1,2 because $G_{l r}^{u p} \supset G_{r}^{u p}$. For the upper bound we write down the tangent space

$$
T_{\left(G_{l r}^{u p} A, A\right)}=\operatorname{Span}_{R}\left(\left(\begin{array}{ccc}
A_{11} U_{11} & \ldots & \sum_{i=1}^{k} A_{1 i} U_{i k} \\
\mathbb{O} & \ldots & \sum_{i=2}^{k} A_{2 i} U_{i k} \\
\ldots & \ldots & \ldots \\
\mathbb{O} & \mathbb{O} & A_{k k} U_{k k}
\end{array}\right),\left(\begin{array}{ccc}
V_{11} A_{11} & \ldots & \sum_{i=1}^{k} V_{1 i} A_{i k} \\
\mathbb{O} & \ldots & \sum_{i=2}^{k} V_{2 i} A_{i k} \\
\ldots & \ldots & \ldots \\
\mathbb{O} & \mathbb{O} & V_{k k} A_{k k}
\end{array}\right)\right)
$$


Thus $f \cdot T_{(\Sigma, A)} \subseteq T_{\left(G_{l r}^{u p} A, A\right)}$ implies $\operatorname{Mat}_{m_{i} \times n_{i}}((f)) \subseteq \operatorname{Span}_{R}\left(V_{i i} A_{i i}+A_{i i} U_{i i}\right)$ for any $i$. But the later means:

$$
f \in \bigcap_{i} \operatorname{ann}\left(T_{\left(\Sigma_{i}, G_{l r}, A_{i i}\right)}^{1}\right) \subseteq \bigcap_{i}\left(\overline{I_{m_{i}}\left(A_{i i}\right)}: \overline{I_{m_{i}-1}\left(A_{i i}\right)}\right) \text {.. }
$$

Example 4.13. $\quad$ i. Suppose the blocks $\left\{A_{q q}\right\}$ are mutually generic so that the ideals ann.coker $\left(A_{q q}\right)$ are relatively prime in the sense: $\prod_{q=1}^{k}$ ann.coker $\left(A_{q q}\right)=\bigcap_{q=1}^{k}$ ann.coker $\left(A_{q q}\right)$. Then we get the equality, $\operatorname{ann}\left(T_{\left(\Sigma, G_{r}^{u p}, A\right)}^{1}\right)=\prod_{q=1}^{k} \operatorname{ann} . \operatorname{coker}\left(A_{q q}\right)$.

ii. Suppose $A_{i j}=\mathbb{O}$ for $1 \leq i<j \leq k$. Then we have the obvious $\operatorname{ann}\left(T_{\left(\Sigma, G_{r}^{u p}, A\right)}^{1}\right)=\bigcap_{i=1}^{k} \operatorname{ann} . \operatorname{coker}\left(A_{i i}\right)$.

iii. Consider the case of one Jordan cell,

$$
A=\left(\begin{array}{ccccc}
a & 1 & 0 & \ldots & \ldots \\
0 & a & 1 & 0 & \ldots \\
\ldots & \ldots & \ldots & \ldots & \ldots \\
0 & \ldots & \ldots & 0 & a
\end{array}\right)
$$

Then, by direct check, ann.coker $(A)=\left(a^{n}\right)$. This realizes the lower bound.

iv. Suppose $A$ is a square matrix, hence $\left\{m_{q}=n_{q}\right\}$, and $\operatorname{det}(A)$ is not a zero divisor in $R$. Suppose, moreover, height $\left(I_{m_{q}-1}\left(A_{q q}\right)\right)>$ 1 for all the blocks. Then we get $(\operatorname{det}(A)) \subseteq \operatorname{ann}\left(T_{\left(\Sigma, G_{r}^{u p}, A\right)}^{1}\right) \subseteq \bigcap_{q}\left(\operatorname{det}\left(A_{q q}\right)\right)$.

4.3.4. Applications to finite determinacy. Theorem 2.6 implies the immediate

Corollary 4.14. (1) If $\operatorname{dim}(R)>\left(n_{i}-m_{i}+1\right)$ for some $i$, then no matrix $A \in M a t_{\left\{m_{i}\right\} \times\left\{n_{j}\right\}}^{u p}(\mathfrak{m})$ is finitely-G $G_{l r}^{u p}-$ determined.

(2) Suppose $R$ satisfies condition (3) and moreover $\operatorname{dim}(R) \geq\left(n_{i}-m_{i}+1\right)$ for all $i$. Then the generic finite-G $G_{r}^{\text {up }}-$ determinacy holds and

$$
\begin{aligned}
& l l_{R}\left(\bigcap_{i} \text { ann.coker }\left(A_{i i}\right)\right)-1 \leq l l_{R}\left(\bigcap_{q} \text { ann.coker }\left(\underline{A} \|_{q}\right)\right) \leq \operatorname{ord}_{G_{r}^{u p}}^{\Sigma} \leq \\
& \leq l l_{R}\left(\bigcap_{q} \text { ann.coker }\left(\left.\underline{A}_{q}\right|_{\mathfrak{m} \cdot R^{j=1}} ^{\sum_{j=1}^{q} n_{j}}\right)\right) \leq l l_{R}\left(\prod_{i} \text { ann.coker }\left(\left.A_{i i}\right|_{\mathfrak{m} \cdot R^{\oplus m}}\right)\right)-1 .
\end{aligned}
$$

4.4. The case of conjugation. Proof of Theorem 2.7. Denote $\Sigma=\operatorname{Mat}_{m \times m}(R)$.

1. First we assume that $R$ is Noetherian. As in the case of congruence, to prove that $\operatorname{ann}\left(T_{\left(\Sigma, G_{\text {conj }}, A\right)}^{1}\right)=\{0\}$ it is enough to prove that $T_{\left(\Sigma, G_{c o n j}, A\right)}^{1}$ is not a torsion on any irreducible component of $\operatorname{Spec}(R)$. Thus we restrict to an irreducible component of $\operatorname{Spec}(R)$ and check $T_{\left(\Sigma, G_{c o n j}, A\right)}^{1}$ at the generic point of $\operatorname{Spec}(R)$, algebraically we localize at the generic ideal $\{0\}$. Therefore we compare the $R_{(0)}$-vector spaces:

$$
M a t_{m \times m}\left(R_{(0)}\right) \quad \text { vs }\left(T_{\left(G_{\text {conj }} A, A\right)}\right)_{(0)}=\operatorname{Span}_{R_{(0)}}\left(U A-A U \mid U \in \operatorname{Mat}_{m \times m}\left(R_{(0)}\right)\right) .
$$

Now the inequality $\left(T_{\left(G_{\text {conj }} A, A\right)}\right)_{(0)} \subsetneq\left(T_{(\Sigma, A)}\right)_{(0)}$ is immediate as $\left(T_{\left(G_{\text {conj }} A, A\right)}\right)_{(0)}$ lies inside the hyperplane $\{B \mid \operatorname{trace}(B)=0\} \subset$ $\left(T_{(\Sigma, A)}\right)_{(0)}$. Therefore $\left(T_{\left(\Sigma, G_{\text {conj }}, A\right)}^{1}\right)_{(0)} \neq\{0\}$.

If $R$ is not Noetherian but satisfies condition $(3)$, then we take the completion $R \stackrel{\hat{\psi}}{\rightarrow} \widehat{R}$. We have ann $\left(\hat{\psi}\left(T_{\left(\Sigma, G_{\text {conj }}, A\right)}^{1}\right)\right)=\{0\}$, thus by Lemma 3.16, $\hat{\psi}\left(\operatorname{ann}\left(T_{\left(\Sigma, G_{\text {conj }}, A\right)}^{1}\right)\right)=\{0\}$. Therefore (as in the case of congruence) we get ann $\left.\left(T_{\left(\Sigma, G_{c o n j}, A\right)}^{1}\right)\right) \subseteq \mathfrak{m}^{\infty}$.

2. Follows immediately from part one and Proposition 3.19.

4.5. Finite determinacy of chains of morphisms. Given a finite collection of free modules, $\left\{F_{i}\right\}_{i \in I}$, of ranks $\left\{m_{i}\right\}_{i \in I}$, and their morphisms, $\phi_{i} \in \operatorname{Hom}_{R}\left(F_{i}, F_{i-1}\right)$, we consider the corresponding chain $\left(F_{\bullet}, \phi_{\bullet}\right)$, not necessarily a complex. We assume $\left\{m_{i+1} \geq m_{i}\right\}$ and omit the terms $F_{i}$ with $m_{i}=0$. The chains $\left(F_{\bullet}, \phi_{\bullet}\right),\left(F_{\bullet}, \psi_{\bullet}\right)$ are called isomorphic if there exist automorphisms $U_{i} \circlearrowright F_{i}$ satisfying: $\psi_{i} \circ U_{i}=U_{i-1} \circ \phi_{i}$.

Definition 4.15. 1. Denote by $\left\{J_{\left(F_{i}, \phi_{i}\right)}\right\}_{i \in I}$ the collection of the largest possible ideals satisfying:

$$
\text { if }\left\{\psi_{i} \equiv \phi_{i} \bmod \left(J_{\left(F_{i}, \phi_{i}\right)}\right)\right\}_{i \in I} \text {, then the chains }\left(F_{\bullet}, \phi_{\bullet}\right) \text { and }\left(F_{\bullet}, \psi_{\bullet}\right) \text { are isomorphic. }
$$

2. Denote by $J_{\left(F_{\bullet}, \phi \bullet\right.}$ the largest possible ideal satisfying:

$$
\text { if }\left\{\psi_{i} \equiv \phi_{i} \bmod \left(J_{\left(F_{\bullet}, \phi_{\bullet}\right)}\right)\right\}_{i \in I} \text {, then the chains }\left(F_{\bullet}, \phi_{\bullet}\right) \text { and }\left(F_{\bullet}, \psi_{\bullet}\right) \text { are isomorphic. }
$$

Lemma 4.16. In both cases the largest object exists and $J_{\left(F_{\bullet}, \phi_{\bullet}\right)}=\bigcap_{i} J_{\left(F_{i}, \phi_{i}\right)}$.

Proof. (We prove the existence in the first case, the second case is similar.)

Call a collection of ideals $\left\{J_{i}\right\}_{i \in I}$ admissible if the conditions $\left\{\psi_{i} \equiv \phi_{i} \bmod \left(J_{i}\right)\right\}_{i \in I}$ imply the isomorphism $\left(F_{\bullet}, \psi_{\bullet}\right) \approx\left(F_{\bullet}, \phi_{\bullet}\right)$. The collection of zero ideals, $\left\{J_{i}=\{0\}\right\}_{i \in I}$, is obviously admissible. Thus it suffices to prove: if $\left\{J_{i}\right\}_{i \in I},\left\{\tilde{J}_{i}\right\}_{i \in I}$ are two admissible collections then the collection $\left\{J_{i}+\tilde{J}_{i}\right\}_{i \in I}$ is admissible.

Suppose $\left\{\psi_{i}=\phi_{i}+\Delta_{i}+\tilde{\Delta}_{i}\right\}_{i \in I}$, where the entries of $\Delta_{i}$ lie in $J_{i}$, while the entries of $\tilde{\Delta}_{i}$ lie in $\tilde{J}_{i}$. Then there exists a collection of automorphisms, $\left\{U_{i} \in A u t_{R}\left(F_{i}\right)\right\}_{i \in I}$ satisfying: $\left\{U_{i} \psi_{i} U_{i}^{-1}=\phi_{i}+U_{i} \tilde{\Delta}_{i} U_{i}^{-1}\right\}_{i \in I}$. Now, the entries of $U_{i} \tilde{\Delta}_{i} U_{i}^{-1}$ still lie in the ideal $\tilde{J}_{i}$. Therefore there exists a collection of automorphisms, $\left\{V_{i} \in A u t_{R}\left(F_{i}\right)\right\}_{i \in I}$ satisfying: $\left\{V_{i} U_{i} \psi_{i} U_{i}^{-1} V_{i}^{-1}=\phi_{i}\right\}_{i \in I}$. The composition $\left\{V_{i} U_{i}\right\}_{i \in I}$ provides the needed automorphism of the chains. 
Finally, both inclusions $J_{\left(F_{\bullet}, \phi_{\bullet}\right)} \subseteq \bigcap_{i} J_{\left(F_{i}, \phi_{i}\right)}$ and $J_{\left(F_{\bullet}, \phi_{\bullet}\right)} \supseteq \bigcap_{i} J_{\left(F_{i}, \phi_{i}\right)}$ are obvious.

Proposition 4.17. Suppose $R$ is Noetherian.

(1) ann.coker $\left(\left.\phi_{i}\right|_{\mathfrak{m} \cdot F_{i}}\right) \subseteq J_{\left(F_{i}, \phi_{i}\right)} \subseteq \overline{I_{m_{i}}\left(\phi_{i}\right)}: \overline{I_{m_{i}-1}\left(\phi_{i}\right)}$.

(2) $\bigcap_{i}$ ann.coker $\left(\left.\phi_{i}\right|_{\mathfrak{m} \cdot F_{i}}\right) \subseteq J_{\left(F_{\bullet}, \phi_{\bullet}\right)} \subseteq \bigcap_{i}\left(\overline{I_{m_{i}}\left(\phi_{i}\right)}: \overline{I_{m_{i}-1}\left(\phi_{i}\right)}\right)$.

This strengthens (quantifies) and generalizes [Cutkosky-Srinivasan, Theorem 5.8].

Proof. 1. The isomorphism of the chains $\left(F_{\bullet}, \phi_{\bullet}\right),\left(F_{\bullet}, \psi_{\bullet}\right)$ implies in particular the left-right equivalence of the representing matrices: $\phi_{i} \stackrel{G_{l r}}{\sim} \psi_{i}$ for each $i$. Thus the embedding $J_{\left(F_{i}, \phi_{i}\right)} \subseteq \overline{I_{m_{i}}\left(\phi_{i}\right)}: \overline{I_{m_{i}-1}\left(\phi_{i}\right)}$ follows immediately from part 3 of Theorem 2.2 and Theorem 3.18 .

To prove the other inclusion observe that if for any $i$ holds: $\phi_{i} \stackrel{G_{r}}{\sim} \psi_{i}$ then $\left(F_{\bullet}, \phi_{\bullet}\right) \stackrel{G_{r}}{\sim}\left(F_{\bullet}, \psi_{\bullet}\right)$. Here $\prod_{i} G_{r}$ acts on $\left\{F_{i}\right\}$ by a collection of automorphisms. Thus the statement follows directly from part 1 of Theorem 2.2 and Theorem 3.18 .

2. This now follows immediately from Lemma 4.16 .

4.6. Criteria for relative determinacy. We start from a preparatory result.

Lemma 4.18. Fix two $R$-modules $N \subseteq M$ and an ideal $J \subset R$.

(1) $\operatorname{ann} J \cdot M / N=\operatorname{ann}(M / N): J$.

(2) $\operatorname{ann} M / N \cdot \operatorname{ann} N / J \cdot N \subseteq \operatorname{ann} M / J \cdot N \subseteq \operatorname{ann} M / N \cap \operatorname{ann} N / J \cdot N \cdot$

Proof. 1. The proof is just the chain of immediate equivalences:

$$
[f \in \operatorname{ann} M / N: J] \text { iff }[f \cdot J \subseteq \text { ann } M / N] \text { iff }[f \cdot J \cdot M \subseteq N] \text { iff } f \in \text { ann } J \cdot M / N \cdot
$$

2. If $f \in \operatorname{ann} M / N$ and $g \in \operatorname{ann} N / J \cdot N$ then $g f \cdot M \subseteq g \cdot N \subseteq J \cdot N$, i.e. $g f \in \operatorname{ann} M / J \cdot N$.

If $f \in \operatorname{ann} M / J \cdot N$ then obviously $f \in \operatorname{ann} M / N$. As $N \subseteq M$ we get also: $f \cdot N \subseteq J \cdot N$, i.e. $f \in$ ann $N / J \cdot N \cdot$

Example 4.19. In each bound of part 2 of the last lemma the equality is realizable.

i. If $\operatorname{dim}(R)$ is large enough and $J \subset R$ is generic for the given $M, N$ then $\operatorname{ann} M / N \cdot \operatorname{ann} N / J \cdot N=\operatorname{ann} M / N \cap a n n N / J \cdot N$, hence part two is an equality.

ii. Let $R=\mathbb{k}[[x, y]]$, with $J=\mathfrak{m}^{p}$ and $N=\left(x^{p}, y^{p}\right) \subset M=R$. Then $J \cdot N=\mathfrak{m}^{2 p}$, ann $N / J \cdot N=\mathfrak{m}^{p}$, ann $M / N=\left(x^{p}, y^{p}\right)$. Therefore:

$$
\left(x^{p}, y^{p}\right)=\operatorname{ann} M / N \cap \operatorname{ann} N / J \cdot N \supsetneq \operatorname{ann} M / J \cdot N=\mathfrak{m}^{2 p}=\operatorname{ann} M / N \cdot \operatorname{ann} N / J \cdot N \cdot
$$

iii. Let $J=N=(f) \subset M=R$. Then $\operatorname{ann} \frac{M}{N} \cdot \operatorname{ann} N / J \cdot N=\operatorname{ann} M / J \cdot N=\left(f^{2}\right) \subsetneq \operatorname{ann} M / N \cap \operatorname{ann} N / J \cdot N=(f)$

This lemma implies the immediate

Corollary 4.20. Let $\Sigma^{(J)}, G^{(I)}$ be as in $\S 2.8$. Suppose $T_{\left(\Sigma^{(J)}, A\right)}=J \cdot T_{(\Sigma, A)}$ and $T_{\left(G^{(I)} A, A\right)}=I \cdot T_{(G A, A)}$. Then:

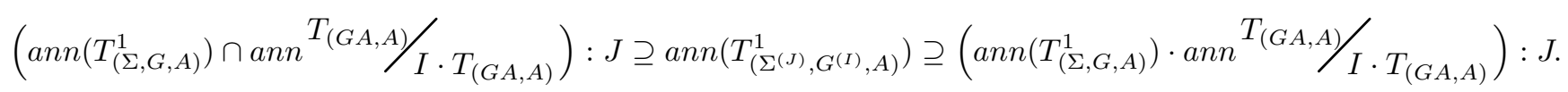

Example 4.21. For $G=G_{r}$ and $I=R$ we get $\operatorname{ann}\left(T_{\left(\Sigma^{(J)}, G_{r}, A\right)}^{1}\right)=\operatorname{ann.coker}(A): J$. If $R$ satisfies the condition $(3)$ and $J^{q} \subseteq$ ann.coker $(A)$ then $A+\operatorname{Mat}_{m \times n}\left(J^{q+k}\right) \subseteq G_{r}^{\left(J^{k}\right)} A$, for any $k>0$. This strengthens and generalizes [Cutkosky-Srinivasan, Theorem 5.2]: "For $R$ a domain and complete with respect to $I_{m}\left(A_{1}\right)$-filtration, there exist $k_{1}, k_{2}$ such that if $A_{1} \equiv A_{2} \bmod \left(I_{m}\left(A_{1}\right)\right)^{j}$ for $j \geq k_{1}$ then $A_{1}=U A_{2} V^{-1}$, with $U=\mathbb{I} \bmod \left(I_{m}\left(A_{1}\right)\right)^{j-k_{2}}, V=\mathbb{I} \bmod \left(I_{m}\left(A_{1}\right)\right)^{j-k_{2}}$."

Example 4.22. Let $A \in \operatorname{Mat}_{m \times m}^{\text {sym }}(\mathfrak{m})$ where $R$ satisfies condition $(3)$ and is Henselian. If $J^{q} \subseteq I_{m}(A): I_{m-1}(A)$ then $\{A\}+$ $M a t_{m \times m}^{s y m}\left(J^{q+k}\right) \subseteq G_{\text {congr }}^{\left(J^{k}\right)} A$ for any $k>0$.

\section{REFERENCES}

[Ahmed-Ruas] I.Ahmed, M.A. Soares-Ruas, Determinacy of Determinantal Varieties, arXiv:1710.11189.

[Arnol'd68] V.I. Arnol'd, Singularities of smooth mappings. Uspehi Mat. Nauk 231968 no. 1, 3-44.

[AGLV-1] V.I. Arnol'd, V.V. Goryunov, O.V. Lyashko, V.A. Vasil'ev, Singularity theory. I. Reprint of the original English edition from the series Encyclopaedia of Mathematical Sciences [ Dynamical systems. VI, Encyclopaedia Math. Sci., 6, Springer, Berlin, 1993]. Springer-Verlag, Berlin, 1998. iv+245 pp. ISBN: 3-540-63711-7

[Beasley-Laffey] L.B. Beasley, T.J. Laffey, Linear operators on matrices: the invariance of rank-k matrices. Linear Algebra Appl. 133 (1990), $175-184$.

[B.K.1] G. Belitski, D. Kerner, Group actions on filtered modules and finite determinacy. Finding large submodules in the orbit by linearization, C. R. Math. Rep. Acad. Sci. Canada Vol. 38 (4) 2016, pp. 113-155 .

[B.K.2] G. Belitski, D. Kerner, Finite determinacy of matrices over local rings.II. Tangent modules to the miniversal deformations for group-actions involving the ring automorphisms, arXiv:1604.06247.

[B.K.3] G. Belitski, D. Kerner, in preparation.

[Birkhoff-MacLane] S. Mac Lane, G. Birkhoff, Algebra. Third edition. Chelsea Publishing Co., New York, 1988. xx+626 pp. 
[Bruce] J.W. Bruce, On families of symmetric matrices. Dedicated to Vladimir I. Arnold on the occasion of his 65th birthday. Mosc. Math. J. 3 (2003), no. $2,335-360,741$

[Bruce-Goryunov-Zakalyukin] J.W. Bruce, V.V. Goryunov, V.M. Zakalyukin, Sectional singularities and geometry of families of planar quadratic forms. Trends in singularities, 83-97, Trends Math., Birkhäuser, Basel, 2002

[Bruce-Tari] J.W. Bruce, F. Tari, On families of square matrices. Proc. London Math. Soc. (3) 89 (2004), no. 3, 738-762.

[Buchsbaum-Eisenbud], D.A. Buchsbaum; D. Eisenbud, Algebra structures for finite free resolutions, and some structure theorems for ideals of codimension 3. Amer. J. Math. 99 (1977), no. 3, 447-485

[Cutkosky-Srinivasan] S.D. Cutkosky, H. Srinivasan, Equivalence and finite determinancy of mappings. J. Algebra 188 (1997), no. 1, 16-57.

[Damon84] J. Damon, The unfolding and determinacy theorems for subgroups of A and K. Mem. Amer. Math. Soc. 50 (1984), no. 306, x+88 pp

[Damon-Pike.1] J. Damon, B. Pike, Solvable Groups, Free Divisors and Nonisolated Matrix Singularities I: Towers of Free Divisors, arXiv:1201.1577, to appear in Annales de l'Institut Fourier.

[Damon-Pike.2] J. Damon, B. Pike, Solvable groups, free divisors and nonisolated matrix singularities II: Vanishing topology. Geom. Topol. 18 (2014), no. 2, 911-962

[Dieudonné] J. Dieudonné, Sur une généralisation du groupe orthogonal à quatre variables. Arch. Math. 1, (1949), $282-287$.

[de Jong-van Straten] T. de Jong, D. van Straten, A deformation theory for nonisolated singularities. Abh. Math. Sem. Univ. Hamburg 60 (1990), $177-208$

[du Plessis1982] A. du Plessis, On the genericity of topologically finitely-determined map-germs. Topology 21 (1982), no. 2, $131-156$.

[du Plessis1983] A. du Plessis, Genericity and smooth finite determinacy. Singularities, Part 1 (Arcata, Calif., 1981), 295-312, Proc. Sympos. Pure Math., 40, Amer. Math. Soc., Providence, RI, 1983

[Eisenbud] D. Eisenbud, Commutative algebra. With a view toward algebraic geometry. Graduate Texts in Mathematics, 150. Springer-Verlag, New York, 1995. xvi+785

[Elkik] R. Elkik, Solutions d'équations à coefficients dans un anneau hensélien. Ann. Sci. École Norm. Sup. (4) 6 (1973), 553-603 (1974)

[Frühbis-Krüger.Zach] A. Frühbis-Krüger, M. Zach, On the Vanishing Topology of Isolated Cohen-Macaulay Codimension 2 Singularities, arXiv: 1501.01915 .

[Goryunov-Mond] V. Goryunov, D. Mond, Tjurina and Milnor numbers of matrix singularities. J. London Math. Soc. (2) 72 (2005), no. 1, $205-224$.

[Goryunov-Zakalyukin] V.V. Goryunov, V.M. Zakalyukin, Simple symmetric matrix singularities and the subgroups of Weyl groups $A_{\mu}$, D $D_{\mu}$, E $E_{\mu}$ Dedicated to Vladimir I. Arnold on the occasion of his 65th birthday. Mosc. Math. J. 3 (2003), no. 2, 507-530, 743-744

[Grandjean00] V. Grandjean, Finite determinacy relative to closed and finitely generated ideals. Manuscripta Math. 103 (2000), no. 3, 313-328.

[Grandjean04] V. Grandjean, Infinite relative determinacy of smooth function germs with transverse isolated singularities and relative Eojasiewicz conditions. J. London Math. Soc. (2) 69 (2004), no. 2, 518-530.

[Greub] W. Greub, Multilinear algebra. Second edition. Universitext. Springer-Verlag, New York-Heidelberg, 1978. vii+294 pp.

[Greuel-Pham] G.-M. Greuel, T.H. Pham Mather-Yau Theorem in Positive Characteristic, arXiv:1308.5153.

[Haslinger] G.-J. Haslinger, Families of Skew-symmetric Matrices Ph.D. Thesis, The University of Liverpool (United Kingdom). 2001.

[Heymans] P. Heymans, Pfaffians and skew-symmetric matrices. Proc. London Math. Soc. (3) 191969 730-768.

[Huneke-Swanson] C. Huneke, I. Swanson, Integral closure of ideals, rings, and modules. London Mathematical Society Lecture Note Series, 336. Cambridge University Press, Cambridge, 2006. xiv +431 pp.

[Jiang] G. Jiang, Functions with non-isolated singularities on Singular Spaces, PhD thesis, Utrecht Netherlands, 1997.

[Kucharz] W. Kucharz, Power series and smooth functions equivalent to a polynomial. Proc. Amer. Math. Soc. 98 (1986), no. 3, 527-533.

[Mather] J.N. Mather, Stability of $C^{\infty}$ mappings. I. The division theorem. Ann. of Math. (2) 871968 89-104. Stability of $C^{\infty}$ mappings. II. Infinitesimal stability implies stability. Ann. of Math. (2) $891969254-291$. Stability of $C^{\infty}$ mappings. III. Finitely determined map-germs. Publ. Inst. Hautes Études Sci. Publ. Math. No. 35, 1968, $279-308$. Stability of $C^{\infty}$ mappings. IV. Classification of stable germs by R-algebras. Publ. Inst. Hautes Études Sci. Publ. Math. No. $371969223-248$. Stability of $C^{\infty}$ mappings. V. Transversality. Advances in Math. 41970 301-336 (1970).

Stability of $C^{\infty}$ mappings. VI: The nice dimensions. Proceedings of Liverpool Singularities-Symposium, I (1969/70), pp. 207-253. Lecture Notes in Math., Vol. 192, Springer, Berlin, 1971

[Li-Pierce] C.-K. Li, S. Pierce, Linear preserver problems. Amer. Math. Monthly 108 (2001), no. 7, 591-605.

[Malgrange] B. Malgrange, Ideals of differentiable functions. Tata Institute of Fundamental Research Studies in Mathematics, No. 3 Tata Institute of Fundamental Research, Bombay; Oxford University Press, London 1967 vii+106 pp

[Molnár] L. Molnár, Selected preserver problems on algebraic structures of linear operators and on function spaces. Lecture Notes in Mathematics, 1895. Springer-Verlag, Berlin, 2007.

[Pellikaan88] R. Pellikaan, Finite determinacy of functions with nonisolated singularities. Proc. London Math. Soc. (3) 57 (1988), no. 2, 357-382.

[Pellikaan90] R. Pellikaan Deformations of hypersurfaces with a one-dimensional singular locus. J. Pure Appl. Algebra 67 (1990), no. 1, 49-71.

[Pereira.10] M.S. Pereira, Variedades Determinantais e Singularidades de Matrizes, PhD thesis, 2010, Universidade de São Paulo, USP, Brasil.

[Pereira.17] M.S. Pereira, Properties of G-Equivalence of Matrices, arXiv:1711.02156.

[Pham] T.H. Pham On finite determinacy of hypersurface singularities and matrices in arbitrary characteristic, PhD thesis, Technische Universität Kaiserslautern, 2016.

[Rudin] W.Rudin, Real and complex analysis. Third edition. McGraw-Hill Book Co., New York, 1987. xiv+416 pp

[Siersma83] D. Siersma, Isolated line singularities. Singularities, Part 2 (Arcata, Calif., 1981), 485-496, Proc. Sympos. Pure Math., 40, Amer. Math. Soc., Providence, RI, 1983

[Siersma00] D. Siersma, The vanishing topology of non isolated singularities. New developments in singularity theory (Cambridge, 2000), 447-472, NATO Sci. Ser. II Math. Phys. Chem., 21, Kluwer Acad. Publ., Dordrecht, 2001

[Teissier] B.Teissier, Variétés polaires. II. Multiplicités polaires, sections planes, et conditions de Whitney. Algebraic geometry (La Rábida, 1981), 314-491, Lecture Notes in Math., 961, Springer, Berlin, 1982.

[Thilliez] V. Thilliez, Infinite determinacy on a closed set for smooth germs with non-isolated singularities, Proc. Amer. Math. Soc. 134 (2006), 1527-1536

[Tougeron] J.C. Tougeron, Idéaux de fonctions différentiables. I. Ann. Inst. Fourier (Grenoble) 181968 fasc. 1, 177-240

[Wall79] C.T.C. Wall, Are maps finitely determined in general? Bull. London Math. Soc. 11 (1979), no. 2, 151-154.

[Wall81] C.T.C. Wall, Finite determinacy of smooth map-germs. Bull. London Math. Soc. 13 (1981), no. 6, 481-539.

[Wilson81] L.-C. Wilson, Infinitely determined map germs. Canad. J. Math. 33 (1981), no. 3, 671-684.

[Wilson82] L.-C. Wilson, Map-germs infinitely determined with respect to right-left equivalence. Pacific J. Math. 102 (1982), no. 1, $235-245$.

Department of Mathematics, Ben Gurion University of the Negev, P.O.B. 653, Be'er Sheva 84105, Israel.

E-mail address: genrich@math.bgu.ac.il

E-mail address: dmitry.kerner@gmail.com 